\title{
THERAPEUTIC APPROACHES IN MITOCHONDRIAL DYSFUNCTION, PROTEOLYSIS, AND STRUCTURAL ALTERATIONS OF DIAPHRAGM AND GASTROCNEMIUS IN RATS WITH CHRONIC HEART FAILURE
}

\begin{tabular}{|c|c|}
\hline Journal: & Journal of Cellular Physiology \\
\hline Manuscript ID & JCP-15-0149.R1 \\
\hline Wiley - Manuscript type: & Original Research Article \\
\hline Date Submitted by the Author: & $\mathrm{n} / \mathrm{a}$ \\
\hline Complete List of Authors: & $\begin{array}{l}\text { Barreiro, Esther; IMIM-Hospital del Mar, UPF, PRBB, CIBERES, } \\
\text { Pulmonology-URMAR } \\
\text { Puig-Vilanova, Ester; Hospital del Mar, Universitat Pompeu Fabra (UPF), } \\
\text { Barcelona Biomedical Research Park (PRBB), Pulmonology Department- } \\
\text { Muscle and Respiratory System Research Unit (URMAR) } \\
\text { Marin-Corral, Judith; Hospital del Mar, PRBB, URMAR } \\
\text { Chacon-Cabrera, Alba; IMIM-Hospital del Mar, UPF, PRBB, CIBERES, } \\
\text { Pulmonology-URMAR } \\
\text { Salazar-Degracia, Anna; IMIM, PRBB, URMAR } \\
\text { Mateu, Xavier; Hospital del Mar, Department of Pharmacy } \\
\text { Puente-Maestu, Luis; Hospital General Gregorio Marañón, Universidad } \\
\text { Complutense de Madrid, Servicio de Neumología } \\
\text { Garcia-Arumi, Elena; Hospital Universitari Vall d'Hebron Institut de Recerca } \\
\text { (VHIR), Universitat Autonoma de Barcelona, Unitat de Patologia } \\
\text { Neuromuscular i Mitocondrial } \\
\text { Andreu, Antoni; Hospital Universitari Vall d'Hebron Institut de Recerca } \\
\text { (VHIR), Universitat Autònoma de Barcelona, Unitat de Patologia } \\
\text { Neuromuscular i Mitocondrial } \\
\text { Molina, Luis; Hospital del Mar-IMIM, Universitat Autònoma de Barcelona, } \\
\text { Cardiology Department, Heart Diseases Biomedical Research Group }\end{array}$ \\
\hline Key Words: & $\begin{array}{l}\text { chronic heart failure-induced cachexia, respiratory and limb muscles, } \\
\text { antioxidants and proteasome inhibitor, proteolysis, mitochondrial } \\
\text { respiratory chain activity and oxygen consumption }\end{array}$ \\
\hline
\end{tabular}

\section{SCHOLARONE ${ }^{\text {IM }}$ \\ Manuscripts}


THERAPEUTIC APPROACHES IN MITOCHONDRIAL DYSFUNCTION, PROTEOLYSIS, AND STRUCTURAL ALTERATIONS OF DIAPHRAGM AND GASTROCNEMIUS IN RATS WITH CHRONIC HEART FAILURE

Esther Barreiro ${ }^{1,2}$, *Ester Puig-Vilanova ${ }^{1}$, *Judith Marin-Corral ${ }^{1}$, Alba ChacónCabrera $^{1,2}$, Anna Salazar-Degracia ${ }^{1,2}$, Xavier Mateu $^{3}$, Luis Puente-Maestu ${ }^{3}$, Elena García-Arumí $^{4,5}$, Antoni L. Andreu ${ }^{4,5}$, Luis Molina ${ }^{6}$

${ }^{1}$ Pulmonology Department-Muscle and Respiratory System Research Unit (URMAR), IMIMHospital del Mar, Parc de Salut Mar, Health and Experimental Sciences Department (CEXS), Universitat Pompeu Fabra (UPF), Barcelona Biomedical Research Park (PRBB), Barcelona, Spain.

${ }^{2}$ Centro de Investigación en Red de Enfermedades Respiratorias (CIBERES), Instituto de Salud Carlos III (ISCIII), Barcelona, Spain.

${ }^{3}$ Servicio de Neumología, Hospital General Gregorio Marañón, Universidad Complutense de Madrid, Madrid, Spain.

${ }^{4}$ Unitat de Patologia Neuromuscular i Mitocondrial, Hospital Universitari Vall d'Hebron Institut de Recerca (VHIR), Universitat Autònoma de Barcelona, Barcelona, Spain.

${ }^{5}$ Centro de Investigación Biomédica en Red de Enfermedades Raras (CIBERER), ISCIII, Barcelona, Spain.

${ }^{6}$ Cardiology Department, Hospital del Mar, Heart Diseases Biomedical Research Group, IMIM, and Department of Medicine, Universitat Autònoma de Barcelona, Barcelona, Spain.

* These two authors have equally contributed to the work.

Corresponding author: Dr. Esther Barreiro, Pulmonology Department, IMIM-Hospital del Mar, PRBB, C/ Dr. Aiguader, 88, Barcelona, E-08003 Spain, Telephone: (+34) 933160385 , Fax: (+34) 933160410 , e-mail: ebarreiro@,imim.es.

Word count: 6,924 words 
Running head: muscle protein catabolism, structure, and function in cardiac cachexia

\section{KEY WORDS:}

- chronic heart failure-induced cachexia

- respiratory and limb muscles

- antioxidants

- proteasome inhibitor

- proteolysis

- mitochondrial respiratory chain activity and oxygen consumption

- muscle structural abnormalities

\section{Number of figures: 7}

Number of tables: 2

\section{Grant information:}

Contract grant sponsor: Instituto de Salud Carlos-III (Spanish Competitiveness Ministry);

Contract grant numbers: CIBERES and FIS 06/1043

Contract grant sponsor: Spanish Respiratory Society (SEPAR); Contract grant number:

SEPAR 2008

Contract grant sponsor: Catalan Foundation of Pulmonology; Contract grant number: FUCAP 2008 


\section{ABSTRACT}

Patients with chronic heart failure (CHF) experience exercise intolerance, fatigue and muscle wasting, which negatively influence their survival. We hypothesized that treatment with either the antioxidant $\mathrm{N}$-acetyl cysteine (NAC) or the proteasome inhibitor bortezomib of rats with monocrotaline-induced CHF may restore inspiratory and limb muscle mass, function, and structure through several molecular mechanisms involved in protein breakdown and metabolism in the diaphragm and gastrocnemius. In these muscles of CHF-cachectic rats with and without treatment with NAC or bortezomib ( $n=10$ /group) and non-cachectic controls, proteolysis (tyrosine release, proteasome activities, ubiquitin-proteasome markers), oxidative stress, inflammation, mitochondrial function, myosin, NF- $\kappa \mathrm{B}$ transcriptional activity, muscle structural abnormalities and fiber morphometry were analyzed together with muscle and cardiac functions. In diaphragm and gastrocnemius of CHF-cachectic rats, tyrosine release, proteasome activity, protein ubiquitination, atrogin-1, MURF-1, NF- $\mathrm{B}$ a activity, oxidative stress, inflammation and structural abnormalities were increased, while muscle and cardiac functions, myosin content, slow- and fast-twitch fiber sizes, and mitochondrial activity were decreased. Concomitant treatment of CHF-cachectic rats with NAC or bortezomib improved protein catabolism, oxidative stress, inflammation, muscle fiber sizes, function and damage, superoxide dismutase and myosin levels, mitochondrial function (complex I, gastrocnemius), cardiac function and decreased NF- $\kappa \mathrm{B}$ transcriptional activity in both muscles. Treatment of CHF-cachectic animals with NAC or bortezomib attenuated the functional (heart, muscles), biological, and structural alterations in muscles. Nonetheless, future studies conducted in actual clinical settings are warranted in order to assess the potential beneficial effects and safety concerns of these pharmacological agents on muscle mass loss and wasting in CHFcachectic patients.

Word count: 249 


\section{INTRODUCTION}

Patients with advanced chronic heart failure (CHF) frequently experience poor exercise tolerance, fatigue and muscle wasting, which are also interrelated (Coats et al, 1994;Dalla et al, 2005; Mancini et al, 1992; Massie et al, 1988). Cachexia, which is characterized by severe body weight and muscle loss together with systemic inflammation (Fearon et al, 2011), is commonly associated with chronic conditions including CHF and cancer(Anker et al, 1997;Anker and Sharma, 2002;Barreiro et al, 2015;Fearon et al, 2011;Gielen et al, 2003; Gielen et al, 2005;Gielen et al, 2012;Lavietes et al, 2004;von Haehling and Anker, 2010). Importantly, cachexia negatively influences the patients' quality of life and survival especially in advanced stages of their disease (Anker et al, 1997;Fearon et al, 2011;von Haehling and Anker, 2010).

Patients with CHF-induced cachexia also exhibited reduced blood flow and poor skeletal muscle metabolism during exercise (Mancini et al, 1992;Massie et al, 1988), and impaired endothelium function of the limbs (Drexler et al, 1992). In other studies, a slow-to-fast switch was also observed in the lower limb muscles of patients with CHF (Lipkin et al, 1988;Mancini et al, 1989;Sullivan et al, 1990), together with reduced muscle oxidative metabolism (Sullivan et al, 1991). Inspiratory muscle weakness, as measured by reduced maximal inspiratory pressure, was also shown in patients with diastolic dysfunction (Lavietes et al, 2004). Interestinglt, exercise training induced several beneficial effects in patients with CHF as demonstrated by the reduction in systemic (Gielen et al, 2003) and local (lower limb muscles) inflammation (Gielen et al, 2005) and proteolytic markers (Gielen et al, 2012) as well as improvements in muscle mitochondrial oxidative capacity (Gielen et al, 2005). In line with this, in several experimental models of $\mathrm{CHF}$, skeletal muscle dysfunction was also shown in the diaphragm (Bowen et al, 2015b;van Hees et al, 2007; van Hees et al, 2008) and 
limb muscles of rats (Bertaglia et al, 2011;Bowen et al, 2015b) and in the respiratory muscles of mice(Bowen et al, 2015a).

Despite that several factors and molecular mechanisms leading to cardiac cachexia have been recently elucidated and share similarities with other muscle wasting conditions (Fearon et al, 2011;Muscaritoli et al, 2006; von Haehling and Anker, 2010), the etiology of muscle dysfunction and wasting still needs to be fully understood in CHF. Insulin resistance and nutritional factors, systemic inflammatory cytokines, and skeletal muscle abnormalities including metabolic alterations underlie cardiac cachexia in patients with CHF (Anker and Sharma, 2002;Dalla et al, 2004;Fearon et al, 2011;Vescovo et al, 1998b;Gielen et al, 2003). Furthermore, in a well-known and validated experimental model of CHF such as that of rats treated with monocrotaline, which is characterized by pulmonary hypertension, right ventricle hypertrophy, and severe muscle wasting in rats (Dalla et al, 2004;Dalla et al, 2005;Vescovo et al, 1998a; Vescovo et al, 1998b;Vescovo et al, 2000;Vescovo et al, 2001), increased levels of oxidative stress were found in the heart (Pichardo et al, 1999) and limb muscles (Bertaglia et al, 2011) of the rodents. Modifications in the expression of contractile proteins such as reduced myosin heavy chain $(\mathrm{MyHC})$ content, myogenin, muscle atrophy, and decreased limb muscle blood flow were also observed in rats with monocrotaline-induced CHF (Vescovo et al, 1998a;Bertaglia et al, 2011).

Interestingly, the increased protein oxidation described in the limb muscles, especially the soleus, of rats with monocrotaline-induced CHF (Bertaglia et al, 2011;Dalla et al, 2005), were partly reverted in response to carvedilol treatment (Dalla et al, 2005). Moreover, in the same muscle of the CHF rats, the number of apoptotic nuclei as measured by the terminal deoxynucleotidyl transferase-mediated dUTP nick-end labeling (TUNEL) assay was increased (Vescovo et al, 1998b), as well as in limb muscles of patients with CHF (Vescovo et al, 2000). In another study, high-salt diet induced heart failure, redox imbalance, atrophy, 
and lower mitochondrial density in the diaphragm and soleus muscles together with increased fatigability, especially of the latter muscle in rats (Bowen et al, 2015b). Importantly, increased levels of several markers of oxidative stress were also found in the blood of patients with CHF, suggesting that antioxidants could exert beneficial effects on these patients (Keith et al, 1998). Indeed, treatment with $\mathrm{N}$-acetyl cysteine (NAC) was shown to improve inspiratory muscle function through several molecular mechanisms in another model of diaphragmatic contractile dysfunction in rats (Barreiro et al, 2005b). On the other hand, pharmacological proteasomal inhibition was also shown to improve in vitro contractile function of the diaphragm together with MyHC content and reduced activity of the proteasome in rats with CHF (van Hees et al, 2008). Nonetheless, it remains to be defined whether protein catabolism signaling, proteolysis, and mitochondrial dysfunction may be attenuated by the action of either NAC or bortezomib in both respiratory and limb muscles in CHF-induced cachexia.

On the basis of this, we hypothesized that treatment with either the antioxidant NAC or the proteasome inhibitor bortezomib of rats with monocrotaline-induced CHF may restore inspiratory and limb muscle mass, function, and structure through several molecular mechanisms involved in protein breakdown and metabolism in the diaphragm and a limb muscle of similar fiber type composition such as the gastrocnemius. Accordingly, in rats with monocrotaline-induced $\mathrm{CHF}$ with and without concomitant treatment with either NAC or bortezomib, the study objectives were as follows: 1) to assess respiratory and peripheral muscle and cardiac functions and 2) to explore protein catabolism and proteolysis signaling, proteasome markers and activity, oxidative stress, inflammation, mitochondrial respiratory chain (MRC) activities and oxygen consumption, and muscle structural abnormalities including muscle fibre sizes in the diaphragm and gastrocnemius. Protein oxidation and inflammatory parameters were also determined in the blood of all the study groups of rats. 


\section{METHODS}

(See the online data supplement for detailed information on all the methodologies used in the study).

\section{Ethical approval and experimental design}

Ethical approval. All animal experiments were conducted in the animal facilities at Parc de Recerca Biomèdica de Barcelona (PRBB, Barcelona, Spain). This controlled study was designed in accordance with the ethical standards on animal experimentation (EU 609/86 CEE, Real Decreto 1201/05 BOE 252, Spain) at PRBB and the Helsinki convention for the use and care of animals. Ethical approval was obtained by the Animal Research Committee (Animal welfare department of Catalonia, EBP-10-1232).

Experimental model of cardiac cachexia. Wistar rats (Interfauna, Barcelona, Spain), of about 8 weeks of age were used for the experiments. Rats were kept under pathogen-free conditions in the animal house facility at PRBB, with a 12:12 h light: dark cycle. In all experimental groups of rats (except for control rodents), CHF was induced by an intraperitoneal injection of monocrotaline $(50 \mathrm{mg} / \mathrm{kg})$ on day 0 , and all experimental groups were studied for a period of 30 days as previously shown (Comini et al, 1996;Dalla et al, 2005;Gardner et al, 2004;Reindel et al, 1990;Vescovo et al, 1998a;Vescovo et al, 2001;Bertaglia et al, 2011;Pichardo et al, 1999). The following groups of rats were studied: 1) non-CHF controls $(\mathrm{N}=10)$, intraperitoneal administration of saline at day 0;2) CHF-cachexia group $(\mathrm{N}=10)$, intraperitoneal administration of monocrotaline at day 0 ; 3) CHF-cachexia+NAC group $(\mathrm{N}=10), \mathrm{CHF}$ induced cachectic rats treated with antioxidant $\mathrm{NAC}$, intraperitoneal administration of monocrotaline at day 0 and oral administration (gavage) $3 \mathrm{mmol} / \mathrm{Kg} / 24$ hours NAC during 30 days as previously described (Barreiro et al, 2005b;Dominguez-Alvarez et al, 2014); and 4) CHF-cachexia+Bortezomib group (N=10), CHF-induced cachectic rats treated with proteasome inhibitor, intraperitoneal administration of monocrotaline on day 0 , 
and intravenous (tail vein) administration of $0.15 \mathrm{mg} / \mathrm{Kg}$ Bortezomib on days $0,6,12,18,24$ and 29 as previously reported ( $\mathrm{Lu}$ et al, 2006) and without anesthesia. The proportions of animals that developed CHF-induced cachexia in the monocrotaline-treated groups were as follows: $53 \%, 41 \%$, and $49 \%$ in $\mathrm{CHF}, \mathrm{CHF}+\mathrm{NAC}$, and $\mathrm{CHF}+$ bortezomib, respectively. Therefore, the actual number of rats treated with monocrotaline on day 0 was higher in order to obtain a final number of 10 animals in each experimental group.

\section{In vivo measurements in the rats}

Body weight. Body weight and food intake were determined every day during the entire duration of the study. Control animals were paired-fed according to the amount of food eaten by the cachectic rodents. CHF-induced cachexia was defined as a body weight gain lower than $15 \%$, which was defined as the median value in CHF-cachexia rats. In all animals, body weight gain was calculated as follows: (final body weight on day 30 - initial body weight on day 0)/ initial body weight on day $0 \times 100$.

Measurements of maximal inspiratory pressure (MIP). The animals were first placed, fully awake, in a polyurethane plethysmographic chamber, the anterior part of which contained a two-way valve (Hans Rudolph, model 2200, Kansas City, MO, USA) following previous studies (Barreiro et al, 2005b;Barreiro et al, 2010a;Dominguez-Alvarez et al, 2014). Both the neck and head of each rat were held by a latex membrane, positioned in close proximity to the valve, while a piston placed in the posterior part of the chamber pushed the animal forward to keep it immobilized. A plastic tube attached to the inspiratory branch of the valve and connected to a pressure transducer (ML 140, ADI Instruments, Castle Hill, Australia) was used to measure inspiratory pressure in all rats. MIP was determined by total occlusion of the inspiratory circuit of the Hans Rudolph valve for 30 seconds, and this maneuver was performed at least twice, with a space of less than 60 seconds between them. MIP was the highest value obtained from the two different measurements and was measured at two 
different time points: day 0 and day 30 in all animals. The percentage of MIP change was calculated as follows: (MIP on day 30 - MIP on day 0 ) / MIP on day $0 \times 100$.

Measurements of limb grip strength. Skeletal muscle force was measured using a classical apparatus (Grip Strength Meter, Bioseb, Chaville, France) specifically designed for grip strength measurements in rodents (rats and mice), consisting of an accurate sensor and an inclined grid below the horizontal, which always remained in a fixed position under the same angle. Briefly, peripheral maximal force was determined in each case as follows. The animal was initially held by the tail above the grid. Immediately afterwards, the rat was moved down until its forelimbs grasped the grid and the animal was then brought to an almost horizontal position. Finally, the rat was pulled out following the axle of the sensor until it released the grid. The entire procedure, which was performed very gently in each animal, lasted for 30 seconds. Measurements were carried out using a highly accurate sensor, together with an electronic device with a sampling rate up to $1,000 \mathrm{~Hz}$, ensuring that the maximum force was perfectly captured and displayed. Readings were expressed in grams (g) and analyzed using specific software (RSIC, Bioseb). In each evaluation of peripheral muscle strength, the highest values of grip force captured during the 30 -second period were, indeed, the actual grip strength measurements employed for the purpose of the study. It should be mentioned that the highest values of grip strength selected in the study corresponded to maneuvers that lasted for 1 second or longer in each case. In each rodent, the procedure was repeated 3 times with a recovery period of 15 minutes between them, and the mean of the highest values of each assessment was used for the purpose of statistical analysis in the present investigation, and the grip strength measurements were determined at two different time points: days 0 and 30 in all animals. These procedures have also been previously published (Barreiro et al, 2010b;Barreiro et al, 2010a;Murphy et al, 2012; Toledo et al, 2011; Vignaud et al, 2010; Whittemore et al, 2003). The percentage of limb strength gain was calculated as 
follows: (grip strength on day 30 - grip strength on day 0 )/ grip strength on day $0 \times 100$.

Echocardiographic evaluation. Echocardiograms were performed by the expert cardiologist in all rats without anestesia in order to avoid any interference with the animal hemodynamics and cardiac parameters as previously shown (Chaves et al, 2001;Johnson, 2008). The animals were firmly held from the back to let the thorax and abdomen widely exposed. The cardiac parameters heart rate and size and pulmonary artery systolic morphology and velocity were assessed in all animals on day 29. Only those monocrotaline-induced animals that developed severe pulmonary hypertension leading to $\mathrm{CHF}$ and cachexia were used for the purpose of the study. The proportions of animals that developed echocardiographic CHF in the monocrotaline-treated groups were as follows: $53 \%, 41 \%$, and $49 \%$ in $\mathrm{CHF}, \mathrm{CHF}+\mathrm{NAC}$, and $\mathrm{CHF}+$ bortezomib, respectively. Animals were diagnosed with CHF following qualitative procedures, based on the appearance of a mesosystolic notch right above the pulmonary valve and before the bifurcation of the pulmonary artery as shown in the Doppler evaluation as previously shown (Kawahara et al, 2005;Otto C, 2013; Johnson, 2008).

\section{Sample collection from the animals}

Rats from all the experimental groups were always sacrificed on day 30. Each rat was inoculated intraperitoneally with $0.3 \mathrm{~mL}$ sodium pentobarbital $(50 \mathrm{mg} / \mathrm{Kg})$. In all cases, the pedal and blink reflexes were evaluated in order to verify total anesthetic depth. At this time, diaphragm and gastrocnemius muscles and blood were obtained from all animals. The weight of both muscles was determined in each animal using a high-precision scale. Tissues immediately placed in cold isolation buffer were used to perform ex vivo measurements (assessment of oxygen consumption using polarographic studies) (Naimi et al, 2011;PuenteMaestu et al, 2009;Puente-Maestu et al, 2012) and tyrosine release (Chacon-Cabrera et al, 2014). Frozen tissues were used for immunoblotting, enzyme-linked immunosorbent assay (ELISA), luciferase reporter gene assay, and mitochondrial enzyme activity assays, while 
paraffin-embedded tissues were used for the assessment of muscle fiber type morphometry, muscle structure abnormalities, muscle inflammatory cells, and TUNEL assay.

\section{Markers of muscle proteolysis}

Tyrosine release. Protein degradation was explored on the basis of the rate of production of free tyrosine from tissue proteins as previously described (Furuno et al, 1990; Tischler et al, 1982; Chacon-Cabrera et al, 2014).

Proteasome activities. Methodologies previously published were followed for proteasome isolation and quantification of the chymotrypsin-like, trypsin-like, and caspase-like activities (van Hees et al, 2008).

Immunoblotting of $1 D$ electrophoresis. Protein levels of proteolytic markers were explored using immunoblotting procedures as previously described (Chacon-Cabrera et al, 2014;Dominguez-Alvarez et al, 2014). In order to validate equal protein loading among various lanes, glyceraldehyde-3-phosphate dehydrogenase (GAPDH) protein was used as the protein loading controls in all the immunoblots.

\section{NF-кB transcriptional activity}

Luciferase reporter gene assay. These procedures followed have been previously published (Chacon-Cabrera et al, 2014).

\section{Muscle and systemic redox balance}

Immunoblotting of $1 D$ electrophoresis in muscle specimens. Protein levels of redox markers were explored using immunoblotting procedures already described above (Chacon-Cabrera et al, 2014; Dominguez-Alvarez et al, 2014).

Identification of carbonylated proteins in muscles using immunoblotting of $2 D$ electrophoresis. Carbonylated proteins were separated and identified in the diaphragm and gastrocnemius muscles, following procedures previously published studies. Identification of carbonylated proteins by mass spectrometry was conducted in the Proteomics Laboratory at 
Universitat Pompeu Fabra following the quality criteria established by ProteoRed standards (Instituto Nacional de Proteómica, Spain) and procedures previously published (Barreiro et al, 2005a;Marin-Corral et al, 2009;Marin-Corral et al, 2010;Barreiro et al, 2013).

Protein carbonyl enzyme-linked immunosorbent assay (ELISA) in blood specimens. EDTA plasma samples and muscle homogenates from all groups of rats were used in this assay (Protein carbonyl enzyme immuno-assay kit, Biocell Corporation Ltd., Papatotoe, New Zealand) following the precise manufacturer's instructions and previous studies (Barreiro et al, 2008; de et al, 2010;Rodriguez et al, 2012).

\section{Muscle and systemic inflammation}

Cytokine enzyme-linked immunosorbent assay (ELISA) measurements in muscle and plasma specimens. Protein levels of the cytokines tumor necrosis factor (TNF)-alpha, interferongamma, interleukin (IL)-1beta and IL-6 were quantified in the muscles (diaphragm and gastrocnemius muscles) and plasma of all study animals using specific sandwich ELISA kits eBiosience, Bender MedSystems, Vienna, Austria) following previously published methodologies (Barreiro et al, 2008; Barreiro et al, 2010b;Fermoselle et al, 2012).

Inflammatory cell counts in muscles. In order to evaluate the presence of inflammatory cells in the muscle fibers, immunohistochemical analyses were conducted in the diaphragms and gastrocnemius muscles of all study groups of rats following similar methodologies published elsewhere (Barreiro et al, 2011; Chacon-Cabrera et al, 2014).

\section{MRC complexes: enzyme activities in muscles}

Homogenization procedures and mitochondrial activities. Mitochondria were isolated from both diaphragm and gastrocnemius muscles and mitochondrial activities were calculated following previously published studies (Fermoselle et al, 2013;Medja et al, 2009).

\section{MRC oxygen consumption in muscles}

Gastrocnemius and diaphragm muscles obtained from rats and rapidly placed into a cold tube 
containing isolation buffer (on ice) were used to assess mitochondrial oxygen consumption following previously published methodologies (Fermoselle et al, 2013;Puente-Maestu et al, 2012).

\section{Myosin content and muscle structural features}

Protein content of myosin heavy chain (MyHC): Immunoblotting of $1 D$ electrophoresis. Protein levels of MyHC were identified using the immunoblotting procedures already described above and the anti-MyHC antibody (clone A4.1025, Upstate-Millipore, Temecula, CA, USA).

Muscle fiber counts and morphometry. On 3-micrometer muscle paraffin-embedded sections from diaphragms and gastrocnemius muscles of all study groups, MyHC-I and -II isoforms were identified using anti-MyHC-I (clone MHC, Biogenesis Inc., Poole, England, UK) and anti-MyHC-II antibodies (clone MY-32, Sigma, Saint Louis, MO), respectively, as published elsewhere (Chacon-Cabrera et al, 2014;Fermoselle et al, 2012;Dominguez-Alvarez et al, 2014).

Structural abnormalities. The area fraction of normal and abnormal muscle was evaluated on 3-micrometer paraffin-embedded sections of the diaphragm and gastrocnemius of all study groups muscles following previously published methodologies (Figures E1 and E2) (ChaconCabrera et al, 2014;Fermoselle et al, 2012;Dominguez-Alvarez et al, 2014).

TUNEL assay. In muscle paraffin-embedded sections, apoptotic nuclei were identified using the TUNEL assay (In Situ Cell Death Detection Kit, POD, Roche Applied Science, Mannheim, Germany, lot number 12469600) in both diaphragms and gastrocnemius muscles from all study groups following precisely the manufacturer's instructions and previously published studies (Barreiro et al, 2011; Chacon-Cabrera et al, 2014).

\section{Statistical analysis}

Statistical analyses were performed using the Statistical Package for the Social Sciences 
(Portable SPSS, PASW Statistics 18.0 version for windows; SPSS Inc, Chicago, IL). Physiological data are presented as mean (standard deviation) in tables whereas biological data are presented as box and whisker plots in figures. For the purpose of the study, two different sets of comparisons were made: i) non-cachectic control rats and all three other experimental groups of rats: CHF-cachexia, CHF-cachexia+NAC, and CHFcachexia+Bortezomib and ii) CHF-cachectic animals and rats with CHF-induced cachexia treated with either NAC (CHF-cachexia+NAC) or bortezomib (CHF-cachexia+Bortezomib). For the two sets of comparisons, potential differences in physiological and all the structural variables among all experimental groups of rats were analyzed using one-way analysis of variance (ANOVA), in which Tukey's post hoc analysis was used to adjust for multiple comparisons. Non-parametric Kruskal-Wallis test with a Mann-Whitney post hoc analysis was used to assess differences of the study biological variables between the experimental groups. The sample size chosen was based on previous studies, where very similar approaches were employed (Barreiro et al, 2005b;Barreiro et al, 2013;Chacon-Cabrera et al, 2014;Fermoselle et al, 2013;Dominguez-Alvarez et al, 2014), and on assumptions of $80 \%$ power to detect an improvement of $>20 \%$ in measured outcomes at a level of significance of $P \leq 0.05$.

\section{RESULTS}

\section{Physiological characteristics of the study animals}

Table 1 illustrates all functional variables of the study groups. All cardiac function parameters analyzed in the study were significantly altered in CHF-cachexia rats compared to the controls (Table 1). Animals were diagnosed with CHF on the basis of the appearance of a mesosystolic notch in the pulmonary valve of the heart (Figure 1), together with a reduction in pulmonary artery systolic velocity and increased heart size (Table 1). Concomitant 
treatment of the cachectic animals with either NAC or bortezomib partly attenuated the cardiac functional alterations, especially heart rate and pulmonary artery systolic velocity (bortezomib) compared to CHF-cachexia rodents, but not the controls as values were similar (Table 1).

At the end of the study period, the percentage of body weight gain, MIP, and limb strength were significantly reduced in CHF-cachexia rats compared to the controls (Table 1). Concomitant treatment with either NAC or bortezomib partially attenuated such a decrease in the cachectic animals compared to CHF-cachexia rats (MIP and grip strength, Table 1) and to the controls (body weight and MIP, reduced compared to control rats) (Table 1). Weights of diaphragm, gastrocnemius, and liver were significantly reduced in CHF rats compared to the controls, whereas lung and heart weights were significantly increased (Table 1). NAC and especially bortezomib partly mitigated muscle and liver weight alterations in the cachectic animals compared to CHF-cachexia rodents and the controls, whereas the weights of heart and lung were greater in CHF-cachexia animals treated with bortezomib than in the controls (Table 1).

\section{Muscle proteolysis}

Tyrosine release and $N F-\kappa B$ activity. In the diaphragm and gastrocnemius muscles of CHFcachectic rats, tyrosine release and NF- $\mathrm{BB}$ transcriptional activity levels were significantly increased compared to non-cachectic control animals (Figures 2A and 2B). Importantly, compared to CHF-cachexia rats, concomitant treatment with either NAC or bortezomib significantly attenuated such an increase in tyrosine release (diaphragm and gastrocnemius) and NF- $\mathrm{kB}$ transcriptional activity levels (gastrocnemius), which were similar to those detected in the control rats (non-significant differences, Figures 2A and 2B).

Proteolytic systems. In the diaphragm, proteasome chymotrypsine-like and trypsine-like activities were significantly increased in $\mathrm{CHF}, \mathrm{CHF}+\mathrm{NAC}$, and $\mathrm{CHF}+$ bortezomib rats 
compared to the controls (Figure 2C). Caspase-like activity was also increased in CHFcachexia rodents compared to control rats, and concomitant treatment with bortezomib, but not NAC, significantly attenuated trypsine-like and caspase-like activities compared to CHFcachexia animals in the diaphragm (Figure 2C). In the gastrocnemius, proteasome chymotrypsine-like, trypsine-like, and caspase-like activities were also significantly increased in CHF-cachexia rats compared to the controls (Figure 2C). CHF rats treated with bortezomib also exhibited an attenuation in the gastrocnemius levels of trypsine-like and caspase-like activities compared to CHF animals, while caspase-like activity levels were significantly greater compared to the controls in CHF-cachexia+NAC rodents (Figure 2C).

In both muscles, levels of $20 \mathrm{~S}$ proteasome subunit $\mathrm{C} 8$ did not significantly differ among study groups (Figures 3A and 3B). No significant differences were observed in protein levels of atrogin-1 in the diaphragm among the study experimental groups (Figure 3C). However, atrogin-1 levels were significantly increased in the gastrocnemius of CHF-cachexia rats compared to the controls, and concomitant treatment with either bortezomib or NAC induced a significant decline in its levels compared to CHF-cachexia rats and even to the controls in those treated with the latter (Figure 3D). In both muscles, levels of MuRF-1 were significantly greater in CHF-cachexia rats than in the controls (Figures $3 \mathrm{E}$ and $3 \mathrm{~F}$ ). Interestingly, concomitant treatment with $\mathrm{NAC}$, but not bortezomib elicited a significant reduction in MuRF-1 protein levels compared to $\mathrm{CHF}-$ cachexia in both muscles and to the controls in the diaphragm (Figures 3E and 3F). In both study muscles, levels of total protein ubiquitination were significantly increased in CHF-cachexia rats compared to the controls (Figures $3 \mathrm{G}$ and $3 \mathrm{H}$ ), while treatment with either NAC or bortezomib induced a significant decline in those levels only in the diaphragm compared to CHF-cachexia rats, but not the controls (similar levels, Figure 3G). 


\section{Muscle and systemic redox balance}

In both muscles, levels of total reactive carbonyls were significantly greater in CHF-cachectic and CHF-cachectic+bortezomib rats than in the controls (Figure 4A). Concomitant treatment with NAC significantly reduced reactive carbonyl levels in the gastrocnemius compared to CHF-cachexia animals but not the controls (similar levels, Figure 4A). Plasma reactive carbonyl levels did not differ among the study groups (Figure 4A, bottom panel). Several glycolytic enzymes and actin were carbonylated in the muscles of all the study rats (Table E1). Levels of MDA-protein adducts did not significantly differ in either the diaphragm or gastrocnemius among the study groups of rats (Figures 4B and 4C). Levels of protein tyrosine nitration were only significantly increased in the diaphragm, but not the gastrocnemius, of both CHF-cachexia and CHF-cachexia+bortezomib animals compared to the controls (Figures 4D and 4E, respectively). Protein levels of SOD2 were significantly lower in both muscles of CHF-cachectic rats compared to the controls (Figures $4 \mathrm{~F}$ and $4 \mathrm{G}$ ). Interestingly, concomitant treatment of CHF-cachectic rodents with either NAC or bortezomib significantly attenuated such a decline in diaphragm and gastrocnemius compared to CHF-cachexia rats but not to the controls (similar levels, Figures $4 \mathrm{~F}$ and $4 \mathrm{G}$ ).

\section{Systemic and muscle inflammation}

Inflammatory cytokines. ELISA levels of TNF-alpha did not significantly differ in any muscle among the study groups (Figure 5A). However, plasma levels of TNF-alpha were significantly increased in all three groups of CHF-cachexia rats compared to the controls (Figure 5A). ELISA interferon-gamma levels were significantly reduced in both muscles and plasma of CHF-cachectic rats compared to the controls (Figure 5B). Compared to CHFcachexia and control rats, concomitant treatment with bortezomib elicited a further decline in interferon-gamma levels in both diaphragm and gastrocnemius muscles, while inducing a significant increase in the plasma compartment (Figure 5B). Moreover, treatment with NAC 
elicited a further decrease in interferon-gamma levels only in the diaphragm of CHF-cachectic rats compared to the controls, whereas in plasma, it induced a significant rise in the levels of this cytokine compared to CHF-cachexia rodents (Figure 5B). Compared to the controls, levels of IL-1 were significantly increased in the diaphragm and plasma of CHF-cachexia rats (Figure 5C). In the gastrocnemius, IL-1 levels were lower in all three groups of CHFcachectic rats compared to the controls (Figure 5C). Concomitant treatment of CHF-cachectic rats with either NAC or bortezomib decreased IL-1 levels in the diaphragm compared to CHF-cachexia but not the controls (similar levels), while no significant differences between groups were seen in plasma (Figure 5C). ELISA levels of IL-6 were significantly decreased in both muscles, but not plasma, of CHF-cachexia rats compared to the controls (Figure 5D). Bortezomib elicited a further decline in IL-6 levels in diaphragm and plasma in CHFcachectic rats compared to the controls (Figure 5D). In gastrocnemius, IL-6 levels were significantly reduced in CHF-cachexia+NAC rodents compared to the controls, while treatment with bortezomib elicited a significant rise in IL-6 levels compared to CHF-cachexia animals, but not the controls (similar levels, Figure 5D).

Inflammatory cells. Inflammatory cell counts although low, were significantly greater in the diaphragms and gastrocnemius muscles of the CHF-cachexia rats compared to the controls (Table 2). Concomitant treatment with either NAC or bortezomib elicited a significant decline in the number of inflammatory cells in both muscles compared to CHF-cachexia rats, but not to the control animals (similar levels in gastrocnemius), while they were significantly higher than in controls in the diaphragm (Table 2).

\section{MRC complexes: enzyme activities}

Activity of citrate synthase (CS) did not differ in any muscle among the study groups of rats (Figure 6A). Levels of complex I activity, as measured by absolute values and as the ratio to CS, were significantly decreased in the diaphragm of all three groups of CHF-cachexia rats 
compared to the controls (Figure 6B, top panels). In the gastrocnemius, complex I activity was also significantly reduced in CHF-cachexia rats compared to the controls, while concomitant treatment with either NAC or bortezomib significantly attenuated such a decrease compared to CHF-cachexia rats (Figure 6B, bottom panels). Activities of complex II and IV, as measured by absolute values and as the ratio to CS, did not differ in any muscle among the study groups (Figures 6C and 6D, respectively).

\section{MRC oxygen consumption}

In the diaphragm, state 3 and state 4 oxygen consumption did not differ among the study groups (Figure 6E, top panels), while in the limb muscle, it was significantly reduced in all three groups of CHF-cachexia rats compared to the controls (Figure 6E, bottom panels). Moreover, cytochrome c oxygen consumption levels were also significantly reduced in the latter muscle of all three groups of CHF-cachexia animals compared to the controls, whereas no significant differences were observed in the respiratory muscle (Figure 6F).

\section{Muscle structural abnormalities}

The content of MyHC was lower in both muscles of CHF-cachexia rats compared to the controls, and concomitant treatment of the cachectic animals with either NAC or bortezomib significantly attenuated such a decrease in diaphragm and gastrocnemius compared to CHFcachexia animals, but not to the controls (similar levels, Figures 7A and 7B). The proportions of slow- and fast-twitch muscle fibers did not differ in any muscle among the study groups of rats (Table 2). Importantly, the sizes of the same muscle fibers were significantly smaller in both muscles of CHF-cachexia rats compared to the controls (Table 2, Figures $7 \mathrm{C}$ and 7D). Concomitant treatment with either NAC or bortezomib attenuated such a reduction in muscle fiber sizes in diaphragm and gastrocnemius compared to CHF-cachexia rodents, and levels were similar to the controls (Table 2, Figures $7 \mathrm{C}$ and 7D). The proportions of total muscle abnormalities were significantly greater in both muscles of CHF-cachexia rats compared to 
the controls (Table 2). Concomitant treatment with either NAC or bortezomib attenuated total muscle abnormalities in the same muscles compared to CHF-cachexia animals and the controls (bortezomib) (Table 2). The number of internal nuclei in both muscles increased in CHF-cachexia rats compared to the controls, and concomitant treatment with bortezomib elicited a further rise in this parameter in both muscles compared to the controls and CHFcachexia animals (gastrocnemius). The number of TUNEL-positive nuclei was significantly greater in both muscles of CHF-cachexia rats compared to the controls, and concomitant treatment with either NAC or bortezomib significantly attenuated such a rise compared to CHF-cachexia rodents, but not to the controls (similar levels, Table 2, Figures 7E and 7F).

\section{DISCUSSION}

In the current study based on the use of a well-validated experimental model of CHF, muscle mass loss and atrophy of the diaphragm and gastrocnemius was demonstrated as measured by the weight and sizes of slow- and fast-twitch muscle fibers along with reduced myosin protein content and increased apoptotic nuclei counts in each type of muscle compared to the control animals. Moreover, a significant decline in the parameters MIP and grip strength was also observed in the CHF-cachectic rats, which is consistent with the previously demonstrated relationship between body weight and both respiratory and limb muscle strength in rats (Barreiro et al, 2010a). In all groups of cachectic rats, the presence of CHF was confirmed as measured by alterations of several echocardiographic parameters including the characteristic mesosystolic notch. From a molecular biology standpoint, enhanced proteolysis together with a rise in several markers of the ubiquitin-proteasome pathway including proteasome activity were detected in the respiratory and limb muscles of the CHF-cachectic rats compared to the controls. An interesting finding was also the increased transcriptional activity observed in the gastrocnemius of the cachectic rodents compared to control animals. Increased protein 
carbonylation as measured by reactive carbonyls was detected in both study muscles of the CHF-cachectic rats together with a decline in SOD2 content. A rise in IL-1beta was only seen in the diaphragm and plasma of the cachectic rodents, while expression of interferon-gamma and IL-6 was reduced in both muscles and blood. Inflammatory cell counts were in general very low, but increased in both muscles of the cachectic rats compared to the controls. A decline in muscle MRC complex I activity and oxygen consumption was observed, the latter only in the limb muscle, of all groups of cachectic animals compared to control rodents. Concomitant treatment of all cachectic animals with either NAC or bortezomib attenuated the decline in body and muscle weights and improved the analyzed structural parameters of both respiratory and limb muscles. Such improvements were translated into better respiratory and limb muscle functions at the end of the study period in the animals receiving the pharmacological treatments compared to CHF-cachectic rats without the therapies. Furthermore, many of the molecular parameters explored in the study also improved in the cachectic animals receiving concomitant treatment with either NAC or bortezomib compared to non-treated cachectic rodents as specifically discussed below.

An interesting finding was that in the CHF-cachectic animals, the decline in force production was observed in both respiratory and peripheral muscle groups, and concomitant treatment with either NAC or bortezomib attenuated such a decline, which reached control levels, especially in the force generated by the limb muscles. Indeed, respiratory muscle weakness, as measured by reduced MIP, was also previously shown in patients with diastolic dysfunction (Lavietes et al, 2004), as well as in experimental models of CHF, in which diaphragm contractile dysfunction was demonstrated in rats (Bowen et al, 2015b; van Hees et al, 2007; van Hees et al, 2008) and mice(Bowen et al, 2015a), as well as in the peripheral muscles of rats (Bertaglia et al, 2011;Bowen et al, 2015b). These findings suggest that cachexia of animals with CHF affects similarly all muscle groups. In keeping with, other 
relevant findings in the investigation were the demonstration that protein catabolism, contractile muscle protein loss, fiber atrophy and MRC activity decline also took place in the main respiratory muscle, the diaphragm, of the CHF-cachectic rats. In fact, for most of the markers involving the loss of muscle mass and function that were analyzed in the study, no striking differences were seen between respiratory and limb muscles. These findings are in line with a previous investigation from our group (Chacon-Cabrera et al, 2014), in which similar structural and molecular features were also observed in the diaphragm and gastrocnemius muscles of lung cancer cachectic mice. Taken together, these results point towards the action of biological mechanisms that similarly induce protein breakdown, contractile protein loss, increased apoptosis, and MRC dysfunction in both respiratory and limb muscles in this experimental model of cardiac cachexia.

Importantly, muscle protein breakdown and NF- $\mathrm{B}$ transcriptional activity significantly declined in the cachectic muscles of the rats treated with the antioxidant or the proteasome inhibitor. Another relevant finding in the study was the improvement seen in both MyHC content and muscle fiber sizes together with a decline in the proportions of muscle structural abnormalities including TUNEL-positive nuclei in both respiratory and limb muscles of the cachectic rats receiving concomitant treatment with either NAC or bortezomib. Taken together, these results suggest that attenuation of catabolism of proteins, among which contractile $\mathrm{MyHC}$ is probably the most relevant and abundant, results in a significant improvement in muscle fiber size and function in both respiratory and limb muscles of the CHF-cachectic rats. These findings are also in agreement with previous investigations, in which diaphragm muscle function and myosin content improved in response to bortezomib in rats bearing congestive heart failure (van Hees et al, 2008).

The proteasome is an energy-dependent proteolytic system that regulates key cellular processes such as NF- $\mathrm{kB}$ signaling, cell metabolism, and DNA repair. Among the different 
pharmacological proteasome inhibitors currently available, bortezomib is the only agent approved for clinical use in patients with multiple myeloma and mantle cell lymphoma (Cvek and Dvorak, 2007). Proteasome was also shown to attenuate muscle mass loss and dysfunction in previous studies and models (Roth et al, 2005;Supinski et al, 2009; van Hees et al, 2008). In a previous study from our group (Chacon-Cabrera et al, 2014), treatment with bortezomib of lung cancer cachectic mice elicited a significant improvement in muscle proteolysis, NF- $\mathrm{kB}$ signaling, and in slow-twitch fiber sizes but not in the function of either respiratory or limb muscles. It is likely that differences between the study models (cancer versus cardiac cachexia) and the metabolic rate of the animals used in each investigation may account for the discrepancies observed among (Chacon-Cabrera et al, 2014;Supinski et al, 2009; van Hees et al, 2008). Another interesting finding in the study was the improvement seen in the gastrocnemius muscle weight but not the respiratory muscle or total body weight in the cachectic rats treated with bortezomib. These findings are also in line with those previously shown in the model of lung cancer cachexia in mice, in which total body weight did not improve in response to proteasomal inhibition (Chacon-Cabrera et al, 2014). Additionally, bortezomib also induced a significant increase in SOD2 content in both muscles of animals with CHF-cachexia. In fact, these findings were similarly encountered in diaphragm and gastrocnemius muscles of mice with lung cancer cachexia, probably through reduction of NF- $\mathrm{kB}$ transcriptional activity (Chacon-Cabrera et al, 2014).

The antioxidant NAC and other antioxidants scavenge reactive oxygen species (ROS), thus, they attenuate oxidative stress and protein oxidation levels in skeletal muscles that partly contribute to enhancing muscle function (Barreiro et al, 2005b;Hida et al, 1996;Khawli and Reid, 1994;Koechlin et al, 2004a;Reid et al, 1994;Shindoh et al, 1990;Van et al, 1992a). The improvement in muscle contractile function observed in different experimental models and settings (Khawli and Reid, 1994;Shindoh et al, 1990;Koechlin et al, 2004b;Barreiro et al, 
2005b;Hida et al, 1996) suggests that ROS play a paramount role in muscle function and contractile performance. In the current investigation, the antioxidant NAC elicited a decline in oxidative stress parameters, a rise in SOD2 protein content, as also demonstrated in a former investigation (Barreiro et al, 2005b), together with an improvement in muscle protein catabolism, muscle structural abnormalities, and in the size of slow- and fast-twitch fibers in both study muscles. Furthermore, a rise in protein levels of MyHC was also seen in response to concomitant treatment with either NAC or bortezomib in both respiratory and limb muscles in the CHF-cachectic rats. It is likely that increased oxidation of contractile MyHC renders the protein more prone to degradation as shown to occur in other models (Fermoselle et al, 2012;Marin-Corral et al, 2009).

Importantly, the MRC complex I activity was reduced in the diaphragm and gastrocnemius muscles as measured by both absolute and the ratio to CS values in CHFcachectic rats. Additionally, cytochrome c oxidase oxygen consumption was decreased only in the limb muscle of the cachectic rodents. Concomitant treatment of the animals with either NAC or bortezomib elicited a significant improvement in complex I activity only in the gastrocnemius muscle. These findings are partly in agreement with a former investigation (Fermoselle et al, 2013), in which the activities of complex I, II, and IV were also decreased in the respiratory and limb muscles of lung cancer cachectic mice and treatment with NAC or bortezomib also elicited an improvement in those parameters. Interestingly, NAC may have also acted as a direct antioxidant within the skeletal muscle fibers by eliciting the protection of specific cellular sites such as the mitochondria proteins from oxidative damage as shown to occur in other models (Barreiro et al, 2005b;Khawli and Reid, 1994;Reid et al, 1994;Reilly et al, 1991; Shindoh et al, 1990; Van et al, 1992b).

Neither NAC nor bortezomib modified oxygen consumption in the study muscles of the CHF-cachectic rats. Moreover, oxygen consumption was decreased in the three groups of 
CHF-cachexia rats only in the gastrocnemius, suggesting that the activity of the muscle may play a relevant role in these findings as opposed to the diaphragm, which must remain active throughout the entire duration of the study. Moreover, differences in the metabolic rates of the study muscles may also account for the differences in the activity of the MRC complexes ans oxygen consumption (Fermoselle et al, 2013). Interestingly, the diaphragm muscle rather than the gastrocnemius exhibited a greater magnitude of the MRC complexes analyzed in the current study. Somehow, this was an expected finding and in line with previous results from our group obtained in muscles from cancer cachectic mice (Fermoselle et al, 2013).

The activity of CS enzyme did not differ in either respiratory or limb muscles between the different study groups of rats. As in previous investigations (Fermoselle et al, 2013;Kavazis et al, 2009;Medja et al, 2009), the activity of CS enzyme was used as a marker of mitochondrial content in the muscle preparations. This finding imply that the decline observed in MRC complex I activity was likely the result of alterations taking place in the enzyme active site than in mitochondrial content within the respiratory and limb muscles of the CHF-cachectic rats.

\section{Study critique}

A potential limitation is related to the eventual translation of the study results to clinical settings of patients with cardiac cachexia derived from the current findings obtained in an animal model of CHF. Nonetheless, the current animal model of CHF-induced cachexia has been previously well-validated in the literature (Dalla et al, 2004;Dalla et al, 2005;Vescovo et al, 1998a;Vescovo et al, 1998b;Vescovo et al, 2000;Vescovo et al, 2001;Bertaglia et al, 2011;Pichardo et al, 1999) as animals show specific pathophysiological and biological features of cardiac cachexia in their muscles that resemble those observed in the patients. Moreover, the pharmacological strategies, although already in use for other clinical purposes, tested in the study for the specific treatment of CHF-induced cachexia require the use of an in 
vivo experimental model before any implementation can be made in actual clinical settings. Another potential limitation is related to the lack of measurements of body composition using dual-energy X-ray absorptiometry (DEXA) procedures. Unfortunately, these methodologies were not available at the time of performing the rat experiments. Nonetheless, as animals were all young (eight weeks), the influence of adipose tissue on final body changes was most likely irrelevant.

Another potential limitation is the lack of in vitro contractility studies of the muscles. As measurements of both MIP and grip strength were obtained at two time-points (baseline and at the end of the study period) on the same animals from all experimental groups, noninvasive well-validated methodologies were chosen in this study.

\section{Conclusions}

We conclude that in this experimental model of CHF-cachexia, the decline in both respiratory and peripheral muscle functions, major contractile proteins and mitochondrial oxygen consumption, enhanced protein catabolism and fiber atrophy, and increased muscle structural alterations, including apoptosis were probably signaled by $\mathrm{NF}-\kappa \mathrm{B}$ transcriptional activity and triggered by oxidative stress but not inflammation. Concomitant treatment of the cachectic animals with either NAC or bortezomib elicited an attenuation of those mechanisms together with a restoration of cardiac function parameters and those of respiratory and limb muscles. Despite the relevance of these findings, future studies conducted in actual clinical settings are warranted in order to assess the potential beneficial effects and safety concerns of these pharmacological agents on muscle mass loss and wasting in CHF-cachectic patients. 


\title{
ACKNOWLEDGEMENTS
}

The authors are grateful to Ms. Alba Simats and Marina Sabaté-Brescó (MSc) for their help with part of the data presentation and to Dr. Juan Martin-Caballero and Mr. Francisco Sanchez for their help and support with the animal experiments. We also gratefully acknowledge the advice provided by Mr. Sergi Mojal (professional statistician) with the statistical analyses and graphical representation of all the study results.

\section{Competing interests declared by all the authors: None.}

\begin{abstract}
AUTHORS' CONTRIBUTIONS: Conception and design: EB; Animal experiments, echocardiographic assessment, and sample collection: EPV, JMC, LM; Molecular biology analyses: EPV, JMC, ACC, ASD; Mitochondrial function assessment: EPV, LPM, EGA, ALA; Statistical analyses and data interpretation and presentation: EPV, ACC, XM, EB; manuscript drafting and intellectual input: EB, EPV, LM, EGA, ALA, LPM; Manuscript writing final version: EB.
\end{abstract}




\section{Literature Cited}

Anker SD, Ponikowski P, Varney S, Chua TP, Clark AL, Webb-Peploe KM, Harrington D, Kox WJ, Poole-Wilson PA, Coats AJ (1997). Wasting as independent risk factor for mortality in chronic heart failure. Lancet 349:1050-1053.

Anker SD, Sharma R (2002). The syndrome of cardiac cachexia. Int J Cardiol 85:51-66.

Barreiro E, Bustamante V, Cejudo P, Galdiz JB, Gea J, de LP, Martinez-Llorens J, Ortega F, Puente-Maestu L, Roca J, Rodriguez-Gonzalez Moro JM (2015). Guidelines for the evaluation and treatment of muscle dysfunction in patients with chronic obstructive pulmonary disease. Arch Bronconeumol 51:384-395.

Barreiro E, Fermoselle C, Mateu-Jimenez M, Sanchez-Font A, Pijuan L, Gea J, Curull V (2013). Oxidative stress and inflammation in the normal airways and blood of patients with lung cancer and COPD. Free Radic Biol Med 65:859-871.

Barreiro E, Ferrer D, Sanchez F, Minguella J, Marin-Corral J, Martinez-Llorens J, Lloreta J, Gea J (2011). Inflammatory cells and apoptosis in respiratory and limb muscles of patients with COPD. J Appl Physiol 111:808-817.

Barreiro E, Gea J, Di FM, Kriazhev L, James S, Hussain SN (2005a). Protein carbonyl formation in the diaphragm. Am J Respir Cell Mol Biol 32:9-17.

Barreiro E, Marin-Corral J, Sanchez F, Mielgo V, Alvarez FJ, Galdiz JB, Gea J (2010a). Reference values of respiratory and peripheral muscle function in rats. J Anim Physiol Anim Nutr (Berl) 94:e393-e401.

Barreiro E, Peinado VI, Galdiz JB, Ferrer E, Marin-Corral J, Sanchez F, Gea J, Barbera JA (2010b). Cigarette smoke-induced oxidative stress: A role in chronic obstructive pulmonary disease skeletal muscle dysfunction. Am J Respir Crit Care Med 182:477-488.

Barreiro E, Sanchez D, Galdiz JB, Hussain SN, Gea J (2005b). N-acetylcysteine increases manganese superoxide dismutase activity in septic rat diaphragms. Eur Respir J 26:10321039.

Barreiro E, Schols AM, Polkey MI, Galdiz JB, Gosker HR, Swallow EB, Coronell C, Gea J (2008). Cytokine profile in quadriceps muscles of patients with severe COPD. Thorax 63:100107.

Bertaglia RS, Reissler J, Lopes FS, Cavalcante WL, Carani FR, Padovani CR, Rodrigues SA, Cigogna AC, Carvalho RF, Fernandes AA, Gallacci M, Silva MD (2011). Differential morphofunctional characteristics and gene expression in fast and slow muscle of rats with monocrotaline-induced heart failure. J Mol Histol 42:205-215.

Bowen TS, Mangner N, Werner S, Glaser S, Kullnick Y, Schrepper A, Doenst T, Oberbach A, Linke A, Steil L, Schuler G, Adams V (2015a). Diaphragm muscle weakness in mice is early-onset post-myocardial infarction and associated with elevated protein oxidation. J Appl Physiol (1985 ) 118:11-19.

Bowen TS, Rolim NP, Fischer T, Baekkerud FH, Medeiros A, Werner S, Bronstad E, Rognmo O, Mangner N, Linke A, Schuler G, Silva GJ, Wisloff U, Adams V (2015b). Heart 
failure with preserved ejection fraction induces molecular, mitochondrial, histological, and functional alterations in rat respiratory and limb skeletal muscle. Eur J Heart Fail 17:263-272.

Chacon-Cabrera A, Fermoselle C, Urtreger AJ, Mateu-Jimenez M, Diament MJ, de Kier Joffe ED, Sandri M, Barreiro E (2014). Pharmacological strategies in lung cancer-induced cachexia: effects on muscle proteolysis, autophagy, structure, and weakness. J Cell Physiol 229:1660-1672.

Chaves AA, Weinstein DM, Bauer JA (2001). Non-invasive echocardiographic studies in mice: influence of anesthetic regimen. Life Sci 69:213-222.

Coats AJ, Clark AL, Piepoli M, Volterrani M, Poole-Wilson PA (1994). Symptoms and quality of life in heart failure: the muscle hypothesis. Br Heart J 72:S36-S39.

Comini L, Gaia G, Curello S, Ceconi C, Pasini E, Benigno M, Bachetti T, Ferrari R (1996). Right heart failure chronically stimulates heat shock protein 72 in heart and liver but not in other tissues. Cardiovasc Res 31:882-890.

Cvek B, Dvorak Z (2007). Targeting of nuclear factor-kappaB and proteasome by dithiocarbamate complexes with metals. Curr Pharm Des 13:3155-3167.

Dalla LL, Ravara B, Gobbo V, Danieli BD, Germinario E, Angelini A, Vescovo G (2005). Skeletal muscle myofibrillar protein oxidation in heart failure and the protective effect of Carvedilol. J Mol Cell Cardiol 38:803-807.

Dalla LL, Ravara B, Volterrani M, Gobbo V, Della BM, Angelini A, Danieli BD, Germinario E, Vescovo G (2004). Beneficial effects of GH/IGF-1 on skeletal muscle atrophy and function in experimental heart failure. Am J Physiol Cell Physiol 286:C138-C144.

de BJ, Barreiro E, Romieu I, Mendez M, Gomez FP, Balcells E, Ferrer J, Orozco-Levi M, Gea J, Anto JM, Garcia-Aymerich J (2010). Dietary modulation of oxidative stress in chronic obstructive pulmonary disease patients. Free Radic Res 44:1296-1303.

Dominguez-Alvarez M, Sabate-Bresco M, Vila-Ubach M, Galdiz JB, Alvarez FJ, Casadevall C, Gea J, Barreiro E (2014). Molecular and physiological events in respiratory muscles and blood of rats exposed to inspiratory threshold loading. Transl Res 163:478-493.

Drexler H, Hayoz D, Munzel T, Hornig B, Just H, Brunner HR, Zelis R (1992). Endothelial function in chronic congestive heart failure. Am J Cardiol 69:1596-1601.

Fearon K, Strasser F, Anker SD, Bosaeus I, Bruera E, Fainsinger RL, Jatoi A, Loprinzi C, MacDonald N, Mantovani G, Davis M, Muscaritoli M, Ottery F, Radbruch L, Ravasco P, Walsh D, Wilcock A, Kaasa S, Baracos VE (2011). Definition and classification of cancer cachexia: an international consensus. Lancet Oncol 12:489-495.

Fermoselle C, Garcia-Arumi E, Puig-Vilanova E, Andreu AL, Urtreger AJ, de Kier Joffe ED, Tejedor A, Puente-Maestu L, Barreiro E (2013). Mitochondrial dysfunction and therapeutic approaches in respiratory and limb muscles of cancer cachectic mice. Exp Physiol 98:13491365.

Fermoselle C, Rabinovich R, Ausin P, Puig-Vilanova E, Coronell C, Sanchez F, Roca J, Gea J, Barreiro E (2012). Does oxidative stress modulate limb muscle atrophy in severe COPD 
patients? Eur Respir J 40:851-862.

Furuno K, Goodman MN, Goldberg AL (1990). Role of different proteolytic systems in the degradation of muscle proteins during denervation atrophy. J Biol Chem 265:8550-8557.

Gardner SY, McGee JK, Kodavanti UP, Ledbetter A, Everitt JI, Winsett DW, Doerfler DL, Costa DL (2004). Emission-particle-induced ventilatory abnormalities in a rat model of pulmonary hypertension. Environ Health Perspect 112:872-878.

Gielen S, Adams V, Linke A, Erbs S, Mobius-Winkler S, Schubert A, Schuler G, Hambrecht $\mathrm{R}$ (2005). Exercise training in chronic heart failure: correlation between reduced local inflammation and improved oxidative capacity in the skeletal muscle. Eur J Cardiovasc Prev Rehabil 12:393-400.

Gielen S, Adams V, Mobius-Winkler S, Linke A, Erbs S, Yu J, Kempf W, Schubert A, Schuler G, Hambrecht R (2003). Anti-inflammatory effects of exercise training in the skeletal muscle of patients with chronic heart failure. J Am Coll Cardiol 42:861-868.

Gielen S, Sandri M, Kozarez I, Kratzsch J, Teupser D, Thiery J, Erbs S, Mangner N, Lenk K, Hambrecht R, Schuler G, Adams V (2012). Exercise training attenuates MuRF-1 expression in the skeletal muscle of patients with chronic heart failure independent of age: the randomized Leipzig Exercise Intervention in Chronic Heart Failure and Aging catabolism study. Circulation 125:2716-2727.

Hida W, Shindoh C, Satoh J, Sagara M, Kikuchi Y, Toyota T, Shirato K (1996). Nacetylcysteine inhibits loss of diaphragm function in streptozotocin-treated rats. Am J Respir Crit Care Med 153:1875-1879.

Johnson K (2008). Introduction to rodent cardiac imaging. ILAR J 49:27-34.

Kavazis AN, Talbert EE, Smuder AJ, Hudson MB, Nelson WB, Powers SK (2009). Mechanical ventilation induces diaphragmatic mitochondrial dysfunction and increased oxidant production. Free Radic Biol Med 46:842-850.

Kawahara Y, Tanonaka K, Daicho T, Nawa M, Oikawa R, Nasa Y, Takeo S (2005). Preferable anesthetic conditions for echocardiographic determination of murine cardiac function. J Pharmacol Sci 99:95-104.

Keith M, Geranmayegan A, Sole MJ, Kurian R, Robinson A, Omran AS, Jeejeebhoy KN (1998). Increased oxidative stress in patients with congestive heart failure. J Am Coll Cardiol 31:1352-1356.

Khawli FA, Reid MB (1994). N-acetylcysteine depresses contractile function and inhibits fatigue of diaphragm in vitro. J Appl Physiol 77:317-324.

Koechlin C, Couillard A, Simar D, Cristol JP, Bellet H, Hayot M, Prefaut C (2004a). Does oxidative stress alter quadriceps endurance in chronic obstructive pulmonary disease? Am J Respir Crit Care Med 169:1022-1027.

Koechlin C, Couillard A, Simar D, Cristol JP, Bellet H, Hayot M, Prefaut C (2004b). Does oxidative stress alter quadriceps endurance in chronic obstructive pulmonary disease? Am J Respir Crit Care Med 169:1022-1027. 
Lavietes MH, Gerula CM, Fless KG, Cherniack NS, Arora RR (2004). Inspiratory muscle weakness in diastolic dysfunction. Chest 126:838-844.

Lipkin DP, Jones DA, Round JM, Poole-Wilson PA (1988). Abnormalities of skeletal muscle in patients with chronic heart failure. Int J Cardiol 18:187-195.

Lu C, Gallegos R, Li P, Xia CQ, Pusalkar S, Uttamsingh V, Nix D, Miwa GT, Gan LS (2006). Investigation of drug-drug interaction potential of bortezomib in vivo in female Sprague-Dawley rats and in vitro in human liver microsomes. Drug Metab Dispos 34:702708.

Mancini DM, Coyle E, Coggan A, Beltz J, Ferraro N, Montain S, Wilson JR (1989). Contribution of intrinsic skeletal muscle changes to 31P NMR skeletal muscle metabolic abnormalities in patients with chronic heart failure. Circulation 80:1338-1346.

Mancini DM, Walter G, Reichek N, Lenkinski R, McCully KK, Mullen JL, Wilson JR (1992). Contribution of skeletal muscle atrophy to exercise intolerance and altered muscle metabolism in heart failure. Circulation 85:1364-1373.

Marin-Corral J, Fontes CC, Pascual-Guardia S, Sanchez F, Olivan M, Argiles JM, Busquets S, Lopez-Soriano FJ, Barreiro E (2010). Redox balance and carbonylated proteins in limb and heart muscles of cachectic rats. Antioxid Redox Signal 12:365-380.

Marin-Corral J, Minguella J, Ramirez-Sarmiento AL, Hussain SN, Gea J, Barreiro E (2009). Oxidised proteins and superoxide anion production in the diaphragm of severe COPD patients. Eur Respir J 33:1309-1319.

Massie BM, Conway M, Rajagopalan B, Yonge R, Frostick S, Ledingham J, Sleight P, Radda G (1988). Skeletal muscle metabolism during exercise under ischemic conditions in congestive heart failure. Evidence for abnormalities unrelated to blood flow. Circulation 78:320-326.

Medja F, Allouche S, Frachon P, Jardel C, Malgat M, Mousson de CB, Slama A, Lunardi J, Mazat JP, Lombes A (2009). Development and implementation of standardized respiratory chain spectrophotometric assays for clinical diagnosis. Mitochondrion 9:331-339.

Murphy KT, Chee A, Trieu J, Naim T, Lynch GS (2012). Importance of functional and metabolic impairments in the characterization of the C-26 murine model of cancer cachexia. Dis Model Mech 5:533-545.

Muscaritoli M, Bossola M, Aversa Z, Bellantone R, Rossi FF (2006). Prevention and treatment of cancer cachexia: new insights into an old problem. Eur J Cancer 42:31-41.

Naimi AI, Bourbeau J, Perrault H, Baril J, Wright-Paradis C, Rossi A, Taivassalo T, Sheel AW, Rabol R, Dela F, Boushel R (2011). Altered mitochondrial regulation in quadriceps muscles of patients with COPD. Clin Physiol Funct Imaging 31:124-131.

Otto C (2013). Cardiomyopathies, hypertensive and pulmonary heart disease. Textbook of clinical ecocardiography.Elsevier Health Sciences. p. 250-251.

Pichardo J, Palace V, Farahmand F, Singal PK (1999). Myocardial oxidative stress changes during compensated right heart failure in rats. Mol Cell Biochem 196:51-57. 
Puente-Maestu L, Perez-Parra J, Godoy R, Moreno N, Tejedor A, Gonzalez-Aragoneses F, Bravo JL, Alvarez FV, Camano S, Agusti A (2009). Abnormal mitochondrial function in locomotor and respiratory muscles of COPD patients. Eur Respir J 33:1045-1052.

Puente-Maestu L, Tejedor A, Lazaro A, de MJ, Alvarez-Sala L, Gonzalez-Aragoneses F, Simon C, Agusti A (2012). Site of mitochondrial reactive oxygen species production in skeletal muscle of chronic obstructive pulmonary disease and its relationship with exercise oxidative stress. Am J Respir Cell Mol Biol 47:358-362.

Reid MB, Stokic DS, Koch SM, Khawli FA, Leis AA (1994). N-acetylcysteine inhibits muscle fatigue in humans. J Clin Invest 94:2468-2474.

Reilly PM, Schiller HJ, Bulkley GB (1991). Pharmacologic approach to tissue injury mediated by free radicals and other reactive oxygen metabolites. Am J Surg 161:488-503.

Reindel JF, Ganey PE, Wagner JG, Slocombe RF, Roth RA (1990). Development of morphologic, hemodynamic, and biochemical changes in lungs of rats given monocrotaline pyrrole. Toxicol Appl Pharmacol 106:179-200.

Rodriguez DA, Kalko S, Puig-Vilanova E, Perez-Olabarria M, Falciani F, Gea J, Cascante M, Barreiro E, Roca J (2012). Muscle and blood redox status after exercise training in severe COPD patients. Free Radic Biol Med 52:88-94.

Roth GA, Moser B, Krenn C, Roth-Walter F, Hetz H, Richter S, Brunner M, Jensen-Jarolim E, Wolner E, Hoetzenecker K, Boltz-Nitulescu G, Ankersmit HJ (2005). Heightened levels of circulating 20S proteasome in critically ill patients. Eur J Clin Invest 35:399-403.

Shindoh C, DiMarco A, Thomas A, Manubay P, Supinski G (1990). Effect of Nacetylcysteine on diaphragm fatigue. J Appl Physiol 68:2107-2113.

Sullivan MJ, Green HJ, Cobb FR (1990). Skeletal muscle biochemistry and histology in ambulatory patients with long-term heart failure. Circulation 81:518-527.

Sullivan MJ, Green HJ, Cobb FR (1991). Altered skeletal muscle metabolic response to exercise in chronic heart failure. Relation to skeletal muscle aerobic enzyme activity. Circulation 84:1597-1607.

Supinski GS, Vanags J, Callahan LA (2009). Effect of proteasome inhibitors on endotoxininduced diaphragm dysfunction. Am J Physiol Lung Cell Mol Physiol 296:L994-L1001.

Tischler ME, Desautels M, Goldberg AL (1982). Does leucine, leucyl-tRNA, or some metabolite of leucine regulate protein synthesis and degradation in skeletal and cardiac muscle? J Biol Chem 257:1613-1621.

Toledo M, Busquets S, Sirisi S, Serpe R, Orpi M, Coutinho J, Martinez R, Lopez-Soriano FJ, Argiles JM (2011). Cancer cachexia: physical activity and muscle force in tumour-bearing rats. Oncol Rep 25:189-193.

van Hees HW, Li YP, Ottenheijm CA, Jin B, Pigmans CJ, Linkels M, Dekhuijzen PN, Heunks LM (2008). Proteasome inhibition improves diaphragm function in congestive heart failure rats. Am J Physiol Lung Cell Mol Physiol 294:L1260-L1268. 
van Hees HW, van der Heijden HF, Ottenheijm CA, Heunks LM, Pigmans CJ, Verheugt FW, Brouwer RM, Dekhuijzen PN (2007). Diaphragm single-fiber weakness and loss of myosin in congestive heart failure rats. Am J Physiol Heart Circ Physiol 293:H819-H828.

Van SC, Boczkowski J, Pasquier C, Du Y, Franzini E, Aubier M (1992a). Effects of Nacetylcysteine on diaphragmatic function and malondialdehyde content in Escherichia coli endotoxemic rats. Am Rev Respir Dis 146:730-734.

Van SC, Boczkowski J, Pasquier C, Du Y, Franzini E, Aubier M (1992b). Effects of Nacetylcysteine on diaphragmatic function and malondialdehyde content in Escherichia coli endotoxemic rats. Am Rev Respir Dis 146:730-734.

Vescovo G, Ambrosio GB, Dalla LL (2001). Apoptosis and changes in contractile protein pattern in the skeletal muscle in heart failure. Acta Physiol Scand 171:305-310.

Vescovo G, Ceconi C, Bernocchi P, Ferrari R, Carraro U, Ambrosio GB, Libera LD (1998a). Skeletal muscle myosin heavy chain expression in rats with monocrotaline-induced cardiac hypertrophy and failure. Relation to blood flow and degree of muscle atrophy. Cardiovasc Res 39:233-241.

Vescovo G, Volterrani M, Zennaro R, Sandri M, Ceconi C, Lorusso R, Ferrari R, Ambrosio GB, Dalla LL (2000). Apoptosis in the skeletal muscle of patients with heart failure: investigation of clinical and biochemical changes. Heart 84:431-437.

Vescovo G, Zennaro R, Sandri M, Carraro U, Leprotti C, Ceconi C, Ambrosio GB, Dalla LL (1998b). Apoptosis of skeletal muscle myofibers and interstitial cells in experimental heart failure. J Mol Cell Cardiol 30:2449-2459.

Vignaud A, Ferry A, Huguet A, Baraibar M, Trollet C, Hyzewicz J, Butler-Browne G, Puymirat J, Gourdon G, Furling D (2010). Progressive skeletal muscle weakness in transgenic mice expressing CTG expansions is associated with the activation of the ubiquitin-proteasome pathway. Neuromuscul Disord 20:319-325.

von Haehling S, Anker SD (2010). Cachexia as a major underestimated and unmet medical need: facts and numbers. J Cachexia Sarcopenia Muscle 1:1-5.

Whittemore LA, Song K, Li X, Aghajanian J, Davies M, Girgenrath S, Hill JJ, Jalenak M, Kelley P, Knight A, Maylor R, O'Hara D, Pearson A, Quazi A, Ryerson S, Tan XY, Tomkinson KN, Veldman GM, Widom A, Wright JF, Wudyka S, Zhao L, Wolfman NM (2003). Inhibition of myostatin in adult mice increases skeletal muscle mass and strength. Biochem Biophys Res Commun 300:965-971. 


\section{FIGURE LEGENDS}

Figure 1: Representative examples of echocardiograms from all the study groups of rats. The mesosystolic notch can be observed in all three groups of CHF-cachectic animals. Definition of abbreviations: CHF, chronic heart failure; NAC, N-acetyl cysteine.

Figures 2A-2B: Standard box plots with median (25th and 75th percentiles) and whiskers (at minimum and maximum values) of tyrosine release $(\mathrm{nmol} / \mathrm{mg} / 2 \mathrm{~h})$ in the diaphragm (top panel A) and gastrocnemius (bottom panel A) muscles of the following experimental groups: Control $\quad(\mathrm{N}=10), \quad$ CHF-cachexia $\quad(\mathrm{N}=10), \quad$ CHF-cachexia+NAC $\quad(\mathrm{N}=10), \quad$ CHFcachexia+Bortezomib ( $\mathrm{N}=10)$. Definition of abbreviations: nmol, nanomol; mg, milligram; $\mathrm{h}$, hour; CHF, chronic heart failure; NAC, N-acetyl cysteine. Outliers in each group are represented as open dots. Statistical significance is represented as follows: *: $p<0.05$, and ***: $\mathrm{p}<0.001$ between any group of CHF-cachexia and control rats, and $\uparrow: \mathrm{p}<0.05$ and $\dagger \dagger \uparrow$ : $\mathrm{p}<0.001$ between any of the pharmacologically treated groups of rats with cachexia and CHFcachexia animals. The dashed line separates both types of comparisons between the groups.

Standard box plots with median (25th and 75th percentiles) and whiskers (at minimum and maximum values) of NF-kB transcriptional activity (firefly RLUs/renilla RLUs) in the gastrocnemius muscle (panel B) of the following experimental groups: Control (N=10), CHFcachexia $(\mathrm{N}=10), \quad$ CHF-cachexia+NAC $\quad(\mathrm{N}=10)$, CHF-cachexia+Bortezomib $\quad(\mathrm{N}=10)$.

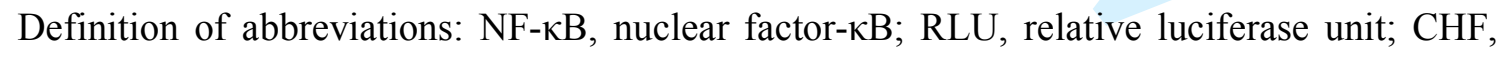
chronic heart failure; NAC, N-acetyl cysteine. Outliers in each group are represented as open dots. Statistical significance is represented as follows: ***: $p<0.001$ between any group of CHF-cachexia and control rats, and $\dagger: p<0.05$ and $\dagger \dagger \dagger: p<0.001$ between any of the pharmacologically treated groups of rats with cachexia and CHF-cachexia animals. The dashed line separates both types of comparisons between the groups.

Figure 2C: Standard box plots with median (25th and 75th percentiles) and whiskers (at 
minimum and maximum values) of chymotrypsin-like activity (nmol/mg) in the diaphragm (top-left panel) and gastrocnemius (bottom-left panel) muscles of the following experimental groups: Control $(\mathrm{N}=10)$, CHF-cachexia $(\mathrm{N}=10)$, CHF-cachexia+NAC $(\mathrm{N}=10)$, CHFcachexia+Bortezomib $(\mathrm{N}=10)$. Definition of abbreviations: nmol, nanomol; mg, milligram; $\mathrm{CHF}$, chronic heart failure; NAC, N-acetyl cysteine. Outliers in each group are represented as open dots. Statistical significance is represented as follows: n.s.: non-significant, $* *: p<0.01$, and $* * *: \mathrm{p}<0.001$, between any group of CHF-cachexia and control rats; and n.s.: nonsignificant between any of the pharmacologically treated groups of rats with cachexia and CHF-cachexia animals. The dashed line separates both types of comparisons between the groups.

Standard box plots with median (25th and 75 th percentiles) and whiskers (at minimum and maximum values) of trypsin-like activity (nmol/mg) in the diaphragm (top-medium panel) and gastrocnemius (bottom-medium panel) muscles of the following experimental groups: Control $\quad(\mathrm{N}=10), \quad$ CHF-cachexia $\quad(\mathrm{N}=10), \quad$ CHF-cachexia+NAC $\quad(\mathrm{N}=10), \quad$ CHFcachexia+Bortezomib ( $\mathrm{N}=10)$. Definition of abbreviations: nmol, nanomole; mg, milligram; $\mathrm{CHF}$, chronic heart failure; NAC, N-acetyl cysteine. Outliers in each group are represented as open dots. Statistical significance is represented as follows: non-significant, *: $p<0.05$, and **: $\mathrm{p}<0.01$ and between any group of CHF-cachexia and control rats; and n.s.: nonsignificant, and $\uparrow: p<0.05$ between any of the pharmacologically treated groups of rats with cachexia and CHF-cachexia animals. The dashed line separates both types of comparisons between the groups.

Standard box plots with median (25th and 75th percentiles) and whiskers (at minimum and maximum values) of caspase-like activity (nmol/mg) in the diaphragm (top-right panel) and gastrocnemius (bottom-right panel) muscles of the following experimental groups: Control $(\mathrm{N}=10)$, CHF-cachexia $(\mathrm{N}=10)$, CHF-cachexia+NAC $(\mathrm{N}=10)$, CHF-cachexia+Bortezomib 
$(\mathrm{N}=10)$. Definition of abbreviations: nmol, nanomol; mg, milligram; CHF, chronic heart failure; NAC, N-acetyl cysteine. Outliers in each group are represented as open dots. Statistical significance is represented as follows: n.s.: non-significant, $* *$ : $p<0.01$, and $* * *$ : $\mathrm{p}<0.001$ between any group of CHF-cachexia and control rats; and n.s.: non-significant, $\uparrow$ : $\mathrm{p}<0.05$, and $\uparrow \dagger: \mathrm{p}<0.01$ between any of the pharmacologically treated groups of rats with cachexia and CHF-cachexia animals. The dashed line separates both types of comparisons between the groups.

Figures 3A-3B: Representative immunoblots of C8-20S in the diaphragm (left panel A) and gastrocnemius (left panel B) muscles of the following experimental groups: Control $(\mathrm{N}=9$ ), CHF-cachexia $(\mathrm{N}=10)$, CHF-cachexia+NAC (diaphragm $\mathrm{N}=5$; gastrocnemius $\mathrm{N}=8$ ), CHFcachexia+Bortezomib (diaphragm $\mathrm{N}=8$; gastrocnemius $\mathrm{N}=9$ ). GAPDH is shown as loading control. Standard box plots with median (25th and 75th percentiles) and whiskers (at minimum and maximum values) of C8-20S protein content in the diaphragm (right panel A) and gastrocnemius (right panel B) muscles, as measured by optical densities in arbitrary units (OD, a.u.). Definition of abbreviations: C8-20S, 20S proteasome subunit C8; KDa, kilodaltons; MW, molecular weights; CHF, chronic heart failure; NAC, N-acetyl cysteine; GAPDH, glyceraldehyde-3-phospate dehydrogenase; OD, optical densities; a.u., arbitrary units. Outliers in each group are represented as open dots. Statistical significance is represented as follows: n.s.: non-significant between any group of CHF-cachexia and control rats; and n.s.: non-significant between any of the pharmacologically treated groups of rats with cachexia and CHF-cachexia animals. The dashed line separates both types of comparisons between the groups.

Figures 3C-3D: Representative immunoblots of atrogin-1 in the diaphragm (left panel C) and gastrocnemius (left panel D) muscles of the following experimental groups: Control ( $\mathrm{N}=9$ ), CHF-cachexia (diaphragm $\mathrm{N}=9$; gastrocnemius $\mathrm{N}=10$ ), CHF-cachexia+NAC (diaphragm 
$\mathrm{N}=5$; gastrocnemius $\mathrm{N}=8$ ), $\mathrm{CHF}$-cachexia+Bortezomib (diaphragm $\mathrm{N}=8$; gastrocnemius $\mathrm{N}=9$ ). GAPDH is shown as the loading control. Standard box plots with median (25th and 75th percentiles) and whiskers (at minimum and maximum values) of Atrogin-1 protein content in the diaphragm (right panel C) and gastrocnemius (right panel D) muscles, as measured by optical densities in arbitrary units (OD, a.u.). Definition of abbreviations: KDa, kilodaltons; MW, molecular weights; CHF, chronic heart failure; NAC, N-acetyl cysteine; GAPDH, glyceraldehyde-3-phospate dehydrogenase; OD, optical densities; a.u., arbitrary units. Outliers in each group are represented as open dots. Statistical significance is represented as follows: n.s.: non-significant, $*$ : $<<0.05$, and $* *: p<0.01$ between any group of CHF-cachexia and control rats, and n.s.: non-significant, $\uparrow: p<0.05$, and $\dagger \dagger: p<0.01$ between any of the pharmacologically treated groups of rats with cachexia and CHF-cachexia animals. The dashed line separates both types of comparisons between the groups.

Figures 3E-3F: Representative immunoblots of MuRF-1 in the diaphragm (left panel E) and gastrocnemius (left panel F) muscles of the following experimental groups: Control $(\mathrm{N}=9$ ), CHF-cachexia (diaphragm $\mathrm{N}=9$; gastrocnemius $\mathrm{N}=10$ ), CHF-cachexia+NAC (diaphragm $\mathrm{N}=5$; gastrocnemius $\mathrm{N}=8$ ), $\mathrm{CHF}$-cachexia+Bortezomib (diaphragm $\mathrm{N}=8$; gastrocnemius $\mathrm{N}=9$ ). GAPDH is shown as the loading control. Standard box plots with median (25th and 75th percentiles) and whiskers (at minimum and maximum values) of MuRF-1 protein content in the diaphragm (right panel E) and gastrocnemius (left panel F) muscles, as measured by optical densities in arbitrary units (OD, a.u.). Definition of abbreviations: MuRF-1, muscle ring finger-1; KDa, kilodaltons; MW, molecular weights; CHF, chronic heart failure; NAC, N-acetyl cysteine; GAPDH, glyceraldehyde-3-phospate dehydrogenase; OD, optical densities; a.u., arbitrary units. Outliers in each group are represented as open dots. Statistical significance is represented as follows: n.s.: non-significant, and $*: p<0.05$ between any group of CHF-cachexia and control rats, and n.s.: non-significant, $\uparrow: p<0.05$, and $\dagger \dagger$ : 
$\mathrm{p}<0.01$ between any of the pharmacologically treated groups of rats with cachexia and CHFcachexia animals. The dashed line separates both types of comparisons between the groups.

Figures 3G-3H: Representative immunoblots of total ubiquitinated proteins in the diaphragm (left panel G) and gastrocnemius (left panel H) muscles of the following experimental groups: Control (N=9), CHF-cachexia $(\mathrm{N}=10)$, CHF-cachexia+NAC (diaphragm $\mathrm{N}=5$; gastrocnemius $\mathrm{N}=8$ ), CHF-cachexia+Bortezomib (diaphragm $\mathrm{N}=8$; gastrocnemius $\mathrm{N}=9$ ). GAPDH is shown as the loading control. Standard box plots with median (25th and 75 th percentiles) and whiskers (at minimum and maximum values) of total ubiquitinated proteins in the diaphragm (right panel G) and gastrocnemius (right panel H) muscles, as measured by optical densities in arbitrary units (OD, a.u.). Definition of abbreviations: MW, molecular weights; KDa, kilodaltons; CHF, chronic heart failure; NAC, N-acetyl cysteine; GAPDH, glyceraldehyde-3phospate dehydrogenase; OD, optical densities; a.u., arbitrary units. Outliers in each group are represented as open dots. Statistical significance is represented as follows: n.s.: nonsignificant, and $*: p<0.05$ between any group of CHF-cachexia and control rats, and n.s.: nonsignificant, $\uparrow: p<0.05$, and $\uparrow \uparrow: p<0.01$ between any of the pharmacologically treated groups of rats with cachexia and CHF-cachexia animals. The dashed line separates both types of comparisons between the groups.

Figure 4A: Standard box plots with median (25th and 75th percentiles) and whiskers (at minimum and maximum values) of total protein carbonylation $(\mathrm{nmol} / \mathrm{mL})$ in the diaphragm (top panel), gastrocnemius (medium panel), and plasma (bottom panel) of the following experimental groups: Control $(\mathrm{N}=10), \mathrm{CHF}-$ cachexia $(\mathrm{N}=10), \mathrm{CHF}-$ cachexia+NAC $(\mathrm{N}=10)$, CHF-cachexia+Bortezomib ( $\mathrm{N}=10)$. Definition of abbreviations: nmol, nanomole; $\mathrm{mL}$, milliliter; CHF, chronic heart failure; NAC, N-acetyl cysteine. Statistical significance is represented as follows: n.s.: non-significant, *: $p<0.05$, and $* *: p<0.01$ between any group of CHF-cachexia and control rats, and n.s.: non-significant, and $\dagger: \mathrm{p}<0.05$ between any of the 
pharmacologically treated groups of rats with cachexia and CHF-cachexia animals. The dashed line separates both types of comparisons between the groups.

Figures 4B-4C: Representative immunoblots of total MDA-protein adducts in the diaphragm (left panel B) and gastrocnemius (left panel C) muscles of the following experimental groups: Control (diaphragm $\mathrm{N}=10$; gastrocnemius $\mathrm{N}=9$ ), CHF-cachexia $(\mathrm{N}=10)$, CHF-cachexia $+\mathrm{NAC}$ (diaphragm $\mathrm{N}=9$; gastrocnemius $\mathrm{N}=8$ ), CHF-cachexia+Bortezomib $(\mathrm{N}=9)$. GAPDH is shown as the loading control. Standard box plots with median (25th and 75 th percentiles) and whiskers (at minimum and maximum values) of total MDA-protein adducts in the diaphragm (right panel B) and gastrocnemius (right panel C) muscles, as measured by optical densities in arbitrary units (OD, a.u.). Definition of abbreviations: MDA, malondialdehyde; MW, molecular weights; KDa, kilodaltons; CHF, chronic heart failure; NAC, N-acetyl cysteine; GAPDH, glyceraldehyde-3-phospate dehydrogenase; OD, optical densities; a.u., arbitrary units. Outliers in each group are represented as open dots. Statistical significance is represented as follows: n.s.: non-significant between any group of CHF-cachexia and control rats, and n.s.: non-significant between any of the pharmacologically treated groups of rats with cachexia and CHF-cachexia animals. The dashed line separates both types of comparisons between the groups.

Figures 4D-4E: Representative immunoblots of total nitrated proteins in the diaphragm (left panel D) and gastrocnemius (left panel E) muscles of the following experimental groups: Control $\quad(\mathrm{N}=10), \quad$ CHF-cachexia $\quad(\mathrm{N}=10), \quad$ CHF-cachexia+NAC $\quad(\mathrm{N}=8), \quad$ CHFcachexia+Bortezomib $(\mathrm{N}=9)$. GAPDH is shown as the loading control. Standard box plots with median (25th and 75 th percentiles) and whiskers (at minimum and maximum values) of total nitrated proteins in the diaphragm (right panel D) and gastrocnemius (right panel E) muscle, as measured by optical densities in arbitrary units (OD, a.u.). Definition of abbreviations: MW, molecular weights; KDa, kilodaltons; CHF, chronic heart failure; NAC, 
$\mathrm{N}$-acetyl cysteine; GAPDH, glyceraldehyde-3-phospate dehydrogenase; OD, optical densities; a.u., arbitrary units. Outliers in each group are represented as open dots. Statistical significance is represented as follows: n.s.: non-significant, and $*$ : $<<0.05$ between any group of CHF-cachexia and control rats, and n.s.: non-significant between any of the pharmacologically treated groups of rats with cachexia and CHF-cachexia animals. The dashed line separates both types of comparisons between the groups

Figures 4F-4G: Representative immunoblots of SOD2 in the diaphragm (left panel F) and gastrocnemius (left panel G) muscles of the following experimental groups: Control $(\mathrm{N}=9)$, CHF-cachexia $(\mathrm{N}=10)$, CHF-cachexia+NAC (diaphragm $\mathrm{N}=5$; gastrocnemius $\mathrm{N}=8$ ), CHFcachexia+Bortezomib (diaphragm $\mathrm{N}=8$; gastrocnemius $\mathrm{N}=9$ ). GAPDH is shown as the loading control. Standard box plots with median (25th and 75th percentiles) and whiskers (at minimum and maximum values) of SOD2 protein content in the diaphragm (right panel F) and gastrocnemius (right panel G) muscles, as measured by optical densities in arbitrary units (OD, a.u.). Definition of abbreviations: SOD2, superoxide dismutase 2, mitochondrial; KDa, kilodaltons; MW, molecular weights; CHF, chronic heart failure; NAC, N-acetyl cysteine; GAPDH, glyceraldehyde-3-phospate dehydrogenase; OD, optical densities; a.u., arbitrary units. Outliers in each group are represented as open dots. Statistical significance is represented as follows: $*$ : $p<0.05$, and $* *: p<0.01$ between any group of CHF-cachexia and control rats, and $\dagger: p<0.05$ between any of the pharmacologically treated groups of rats with cachexia and CHF-cachexia animals. The dashed line separates both types of comparisons between the groups.

Figures 5A-5B: Standard box plots with median (25th and 75th percentiles) and whiskers (at minimum and maximum values) of TNF-alpha $(\mathrm{pg} / \mathrm{mL})$ in the diaphragm (top panel A), gastrocnemius (medium panel A), and plasma (bottom panel A) of the following experimental groups: Control $(\mathrm{N}=10)$, CHF-cachexia $(\mathrm{N}=10)$, CHF-cachexia+NAC $(\mathrm{N}=10)$, CHF- 
cachexia+Bortezomib $(\mathrm{N}=10)$. Definition of abbreviations: pg, picogram; $\mathrm{mL}$, milliliter; $\mathrm{CHF}$, chronic heart failure; NAC, N-acetyl cysteine. Outliers in each group are represented as open dots. Statistical significance is represented as follows: non-significant, n.s.: non-significant, *: $\mathrm{p}<0.05$, and $* * *: \mathrm{p}<0.001$ between any group of CHF-cachexia and control rats, and n.s.: nonsignificant between any of the pharmacologically treated groups of rats with cachexia and CHF-cachexia animals. The dashed line separates both types of comparisons between the groups.

Standard box plots with median (25th and 75 th percentiles) and whiskers (at minimum and maximum values) of interferon-gamma $(\mathrm{pg} / \mathrm{mL})$ in the diaphragm (top panel B), gastrocnemius (medium panel B), and plasma (bottom panel B) of the following experimental groups: Control $(\mathrm{N}=10)$, CHF-cachexia $(\mathrm{N}=10)$, CHF-cachexia+NAC $(\mathrm{N}=10)$, CHFcachexia+Bortezomib (N=10). Definition of abbreviations: pg, picogram; $\mathrm{mL}$, milliliter; $\mathrm{CHF}$, chronic heart failure; NAC, N-acetyl cysteine. Outliers in each group are represented as open dots. Statistical significance is represented as follows: n.s.: non-significant, *: $p<0.05, * *$ : $\mathrm{p}<0.01$, and $* * *: \mathrm{p}<0.001$ between any group of CHF-cachexia and control rats, and n.s.: nonsignificant, $\uparrow: p<0.05, \dagger \uparrow \uparrow: p<0.001$ between any of the pharmacologically treated groups of rats with cachexia and CHF-cachexia animals. The dashed line separates both types of comparisons between the groups.

Figures 5C-5D: Standard box plots with median (25th and 75th percentiles) and whiskers (at minimum and maximum values) of interleukin-1-beta $(\mathrm{pg} / \mathrm{mL})$ in the diaphragm (top panel C), gastrocnemius (medium panel C), and plasma (bottom panel C) of the follow experimental groups: Control $(\mathrm{N}=10), \mathrm{CHF}-$ cachexia $(\mathrm{N}=10), \mathrm{CHF}-$ cachexia+NAC $(\mathrm{N}=10)$, CHF-cachexia+Bortezomib ( $\mathrm{N}=10)$. Definition of abbreviations: pg, picogram; mL, milliliter; $\mathrm{CHF}$, chronic heart failure; NAC, N-acetyl cysteine. Outliers in each group are represented as open dots. Statistical significance is represented as follows: n.s.: non-significant, ${ }^{*}: \mathrm{p}<0.05$, 
**: $\mathrm{p}<0.01$, and ${ }^{* * *}: \mathrm{p}<0.001$ between any group of CHF-cachexia and control rats; and n.s.: non-significant, and $\uparrow: p<0.05$ between any of the pharmacologically treated groups of rats with cachexia and CHF-cachexia animals. The dashed line separates both types of comparisons between the groups.

Standard box plots with median (25th and 75 th percentiles) and whiskers (at minimum and maximum values) of interleukin-6 (pg/mL) in the diaphragm (top panel D), gastrocnemius (medium panel D) and plasma (bottom panel D) of the following experimental groups: Control $\quad(\mathrm{N}=10), \quad$ CHF-cachexia $\quad(\mathrm{N}=10), \quad$ CHF-cachexia+NAC $\quad(\mathrm{N}=10), \quad$ CHFcachexia+Bortezomib ( $\mathrm{N}=10)$. Definition of abbreviations: pg, picogram; $\mathrm{mL}$, milliliter; $\mathrm{CHF}$, chronic heart failure; NAC, N-acetyl cysteine. Outliers in each group are represented as open dots. Statistical significance is represented as follows: n.s.: non-significant, *: $p<0.05, * *$ : $\mathrm{p}<0.01$, and $* * *: \mathrm{p}<0.001$ between any group of CHF-cachexia and control rats; and n.s.: nonsignificant, and $\uparrow \dagger \uparrow: p<0.001$ between any of the pharmacologically treated groups of rats with cachexia and CHF-cachexia animals. The dashed line separates both types of comparisons between the groups.

Figure 6A: Standard box plots with median (25th and 75th percentiles) and whiskers (at minimum and maximum values) of citrate synthase activity (nmol/min/mg) in the diaphragm (top panel) and gastrocnemius (bottom panel) muscles of the following experimental groups: Control $\quad(\mathrm{N}=10), \quad$ CHF-cachexia $\quad(\mathrm{N}=10), \quad$ CHF-cachexia+NAC $\quad(\mathrm{N}=10), \quad$ CHFcachexia+Bortezomib ( $\mathrm{N}=10)$. Definition of abbreviations: nmol, nanomole; min, minute; mg, milligram; CHF, chronic heart failure; NAC, N-acetyl cysteine. Statistical significance is represented as follows: n.s.: non-significant between any group of CHF-cachexia and control rats; and n.s.: non-significant between any of the pharmacologically treated groups of rats with cachexia and CHF-cachexia animals. The dashed line separates both types of comparisons between the groups. 
Figure 6B: Standard box plots with median (25th and 75th percentiles) and whiskers (at minimum and maximum values) of complex I activity (nmol/min/mg) (left panels) and of the ratio of complex I to citrate synthase activities (right panels) in the diaphragm (left- and righttop panels, respectively) and gastrocnemius (left- and right-bottom panels, respectively) muscles of the following experimental groups: Control $(\mathrm{N}=10)$, CHF-cachexia $(\mathrm{N}=10)$, CHFcachexia+NAC $(\mathrm{N}=10)$, CHF-cachexia+Bortezomib $(\mathrm{N}=10)$. Definition of abbreviations: nmol, nanomole; min, minute; mg, milligram; $\mathrm{CHF}$, chronic heart failure; NAC, N-acetyl cysteine. Outliers in each group are represented as open dots. Statistical significance is represented as follows: $*$ : $<<0.05$, and $* *: p<0.01$ between any group of CHF-cachexia and control rats, and $\dagger: p<0.05$, $\dagger \uparrow: p<0.01$, and $\dagger \dagger \uparrow: p<0.001$ between any of the pharmacologically treated groups of rats with cachexia and CHF-cachexia animals. The dashed line separates both types of comparisons between the groups.

Figure 6C: Standard box plots with median (25th and 75th percentiles) and whiskers (at minimum and maximum values) of complex II activity (nmol/min/mg) (left panels) and of the ratio of complex II to citrate synthase activities (right panels) in the diaphragm (left- and right-top panels, respectively) and gastrocnemius (left- and right-bottom panels, respectively) muscles of the following experimental groups: Control $(\mathrm{N}=10)$, CHF-cachexia $(\mathrm{N}=10), \mathrm{CHF}-$ cachexia+NAC $(\mathrm{N}=10)$, CHF-cachexia+Bortezomib $(\mathrm{N}=10)$. Definition of abbreviations: nmol, nanomole; min, minute; mg, milligram; CHF, chronic heart failure; NAC, N-acetyl cysteine. Outliers in each group are represented as open dots. Statistical significance is represented as follows: n.s.: non-significant between any group of CHF-cachexia and control rats, and between any of the pharmacologically treated groups of rats with cachexia and CHFcachexia animals. The dashed line separates both types of comparisons between the groups.

Figure 6D: Standard box plots with median (25th and 75th percentiles) and whiskers (at minimum and maximum values) of complex IV activity (nmol/min/mg) (left panels) and of 
the ratio of complex IV to citrate synthase activities (right panels) in the diaphragm (left- and right-top panels, respectively) and gastrocnemius (left- and right-bottom panels, respectively) muscles of the following experimental groups: Control $(\mathrm{N}=10)$, CHF-cachexia $(\mathrm{N}=10), \mathrm{CHF}-$ cachexia+NAC $(\mathrm{N}=10)$, CHF-cachexia+Bortezomib $(\mathrm{N}=10)$. Definition of abbreviations: nmol, nanomole; min; minute, mg, milligram; CHF, chronic heart failure; NAC, N-acetyl cysteine. Statistical significance is represented as follows: n.s.: non-significant between any group of CHF-cachexia and control rats, and between any of the pharmacologically treated groups of rats with cachexia and CHF-cachexia animals. The dashed line separates both types of comparisons between the groups.

Figure 6E: Standard box plots with median (25th and 75th percentiles) and whiskers (at minimum and maximum values) of state 3 (left panels) and state 4 (right panels) oxygen consumption (nmol $\mathrm{O}_{2} / \mathrm{min} / \mathrm{umg}$ mitochohdria) in the diaphragm (top panels) and gastrocnemius (bottom panels) muscles of the following experimental groups: Control $(\mathrm{N}=10)$, CHF-cachexia $(\mathrm{N}=10)$, CHF-cachexia+NAC $(\mathrm{N}=10)$, CHF-cachexia+Bortezomib $(\mathrm{N}=10)$. Definition of abbreviations: nmol, nanomole; $\mathrm{O}_{2}$, oxygen; min, minute; ug, microgram; CHF, chronic heart failure; NAC, N-acetyl cysteine. Outliers in each group are represented as open dots. Statistical significance is represented as follows: n.s.: nonsignificant, $*: \mathrm{p}<0.05$, and $* * *: \mathrm{p}<0.001$ between any group of CHF-cachexia and control rats, and n.s.: non-significant between any of the pharmacologically treated groups of rats with cachexia and CHF-cachexia animals. The dashed line separates both types of comparisons between the groups.

Figure 6F: Standard box plots with median (25th and 75th percentiles) and whiskers (at minimum and maximum values) of cytochrome $\mathrm{C}$ oxidase oxygen consumption (nmol/min/umg) in the diaphragm (top panel) and gastrocnemius (bottom panel) muscles of the following experimental groups: Control $(\mathrm{N}=10)$, CHF-cachexia $(\mathrm{N}=10)$, CHF- 
cachexia+NAC $(\mathrm{N}=10), \mathrm{CHF}-$ cachexia+Bortezomib $(\mathrm{N}=10)$. Definition of abbreviations: nmol, nanomole; min, minute; ug, microgram; CHF, chronic heart failure; NAC, N-acetyl cysteine. Outliers in each group are represented as open dots. Statistical significance is represented as follows: n.s.: non-significant, $*$ : $p<0.05$, and $* * *: p<0.001$ between any group of CHF-cachexia and control rats, and n.s.: non-significant between any of the pharmacologically treated groups of rats with cachexia and CHF-cachexia animals. The dashed line separates both types of comparisons between the groups.

Figures 7A-7B: Representative immunoblots of MyHC in the diaphragm (left panel A) and gastrocnemius (left panel B) muscles of the following experimental groups: Control (diaphragm $\mathrm{N}=10$; gastrocnemius $\mathrm{N}=9$ ), CHF-cachexia $(\mathrm{N}=9)$, CHF-cachexia+NAC $(\mathrm{N}=8)$, CHF-cachexia+Bortezomib (diaphragm $\mathrm{N}=9$; gastrocnemius $\mathrm{N}=8$ ). GAPDH is shown as the loading control. Standard box plots with median (25th and 75th percentiles) and whiskers (at minimum and maximum values) of MyHC protein content in the diaphragm (right panel A) and gastrocnemius (right panel B) muscles, as measured by optical densities in arbitrary units (OD, a.u.). Definition of abbreviations: MyHC, myosin heavy chain, mitochondrial; KDa, kilodaltons; MW, molecular weights; CHF, chronic heart failure; NAC, N-acetyl cysteine; GAPDH, glyceraldehyde-3-phospate dehydrogenase; OD, optical densities; a.u., arbitrary units. Outliers in each group are represented as open dots. Statistical significance is represented as follows: *: $\mathrm{p}<0.05$ between any group of CHF-cachexia and control rats, and $\uparrow$ : $\mathrm{p}<0.05$ and $\dagger \dagger: \mathrm{p}<0.01$ between any of the pharmacologically treated groups of rats with cachexia and CHF-cachexia animals. The dashed line separates both types of comparisons between the groups.

Figure 7C: Representative examples of stained muscle fibers (x 400) within the diaphragm of the different study groups of rats. Type II fibers were positively stained (brown color) with the corresponding antibody. Note that the two types of fibers were of smaller size in the CHF- 
cachexia rats compared to control rats whereas they were of bigger size in both, CHFcachexia+NAC and CHF-cachexia+Bortezomib groups of treated rats compared to nontreated CHF-cachexia rats (Table 3). Definition of abbreviations: CHF, chronic heart failure; NAC, N-acetyl cysteine.

Figure 7D: Representative examples of stained muscle fibers (x 400) within the gastrocnemius of the different study groups of rats. Type II fibers were positively stained (brown color) with the corresponding antibody. Note that the two types of fibers were of smaller size in the CHF-cachexia rats compared to control rats whereas they were of bigger size in both CHF-cachexia+NAC and CHF-cachexia+Bortezomib groups of animals compared to non-treated CHF-cachexia rats (Table 3). Definition of abbreviations: CHF, chronic heart failure; NAC, N-acetyl cysteine.

Figure 7E: Representative examples of nuclei positively and negatively stained for the TUNEL assay in the diaphragm muscles of the different study groups of rats (x400). Negative nuclei appear in blue (hematoxylin counterstaining, red arrows), while TUNEL-positive nuclei are brown (black arrows). Note that CHF-cachexia rats exhibited greater proportions of TUNEL-positive nuclei than in control rats, whereas CHF-cachexia+NAC and CHFcachexia+Bortezomib groups of rats showed reduced proportions of TUNEL-positive nuclei compared to non-treated CHF-cachexia rats (Table 3). Definition of abbreviations: CHF, chronic heart failure; NAC, N-acetyl cysteine.

Figure 7F: Representative examples of nuclei positively and negatively stained for the TUNEL assay in the gastrocnemius muscles of the different study groups of rats $(\mathrm{x} 400)$. Negative nuclei appear in blue (hematoxylin counterstaining, red arrows), while TUNELpositive nuclei are brown (black arrows). Note that CHF-cachexia rats exhibited greater proportions of TUNEL-positive nuclei than in control rats, whereas CHF-cachexia+NAC and CHF-cachexia+Bortezomib groups of rats showed reduced proportions of TUNEL-positive 
1

2

3

4

5

6

7

8

9

10

11

12

13

14

15

16

17

18

19

20

21

22

23

24

25

26

27

28

29

30

31

32

33

34

35

36

37

38

39

40

41

42

43

44

45

46

47

48

49

50

51

52

53

54

55

56

57

58

59

60

nuclei compared to non-treated CHF-cachexia rats (Table 3). Definition of abbreviations:

CHF, chronic heart failure; NAC, N-acetyl cysteine. 
Table 1. Physiological characteristics of the animal groups at baseline and at the end of the study period (30 days)

\begin{tabular}{|c|c|c|c|c|}
\hline & $\begin{array}{c}\text { Control } \\
n=10 \\
\end{array}$ & $\mathrm{n}=10$ & $\begin{array}{c}\text { CHF-cachexia } \\
\text { + NAC } \\
n=10 \\
\end{array}$ & $\begin{array}{c}\text { CHF-cachexia } \\
\text { + Bortezomib } \\
n=10 \\
\end{array}$ \\
\hline Age (weeks) & 8 & 8 & 8 & 8 \\
\hline \multicolumn{5}{|l|}{ Cardiac parameters } \\
\hline Heart rate $(\mathrm{bpm})$ & $501.5(27.8)$ & $427.25(18.6)^{* * *}$ & $473.67(35.4) \dagger \dagger$ & $485(36.3) \dagger \dagger$ \\
\hline Pulmonary artery systolic velocity (m/s) & $0.84(0.13)$ & $0.62(0.09)^{* * *}$ & $0.68(0.12)$ & $0.84(0.13) \dagger \dagger$ \\
\hline Heart size $(\mathrm{mm})$ & $8.89(1.12)$ & $10.32(0.74)^{* *}$ & $9.98(0.94)$ & $9.50(0.81)$ \\
\hline \multicolumn{5}{|l|}{ Body weight } \\
\hline Baseline (g) & $270.97(11.3)$ & $272.28(21.3)$ & $275.37(11.8)$ & $280.67(7.33)$ \\
\hline End of study (g) & $337.39(15.6)$ & $295.14(32.7)^{* * *}$ & $306.74(20)^{* * *}$ & $305.10(17.11)^{* * *}$ \\
\hline Body weight gain (\%) & $+24.66(4.62)$ & $+8.242(6.03)^{* * *}$ & $+11.33(3.97)^{* * *}$ & $+8.27(4.77)^{* * *}$ \\
\hline \multicolumn{5}{|l|}{ Weights of muscles and organs } \\
\hline Diaphragm weight $(\mathrm{g})$ & $0.85(0.12)$ & $0.77(0.09)^{*}$ & $0.78(0.11)$ & $0.81(0.10)$ \\
\hline Gastrocnemius weight (g) & $1.95(0.14)$ & $1.74(0.14)^{* * *}$ & $1.81(0.19)$ & $1.92(0.22) \dagger \dagger$ \\
\hline Liver weight (g) & $10.34(1.10)$ & $8.23(1.24)^{* * *}$ & $10.96(1.69) \dagger \dagger$ & $11.74(1.34) \dagger \dagger \dagger$ \\
\hline Heart weight (g) & $0.93(0.04)$ & $1.13(0.21)^{*}$ & $1.06(0.17)$ & $1.29(0.17)^{* * *}$ \\
\hline Lung weight (g) & $1.19(0.08)$ & $1.77(0.44)^{*}$ & $1.65(0.23)$ & $2.07(0.32)^{* * *}$ \\
\hline \multicolumn{5}{|l|}{ MIP measurements } \\
\hline Baseline $\left(\mathrm{cm} \mathrm{H}_{2} \mathrm{O}\right)$ & $-11.24(0.8)$ & $-11.64(2.7)$ & $-10.83(4.0)$ & $-10.50(8.44)$ \\
\hline End of study $\left(\mathrm{cm} \mathrm{H}_{2} \mathrm{O}\right)$ & $-15.35(2.1)$ & $-7.64(2.9)^{* * *}$ & $-11.10(4.5)$ & $-10.68(8.57)$ \\
\hline MIP change $(\%)$ & $+36.71(18.6)$ & $-35.08(19.0)^{* * *}$ & $+1.72(6.55)^{* * *, \dagger \dagger \dagger}$ & $+1.81(9.06)^{* *}, \dagger \dagger \dagger$ \\
\hline \multicolumn{5}{|l|}{ Grip strength measurements } \\
\hline Baseline (g) & $5.77(0.83)$ & $6.83(1.42)$ & $5.57(0.59)$ & $5.99(0.77)$ \\
\hline
\end{tabular}


End of study (g)

$+34.88(5.32)$

$+1.46(7.86) * * *$

$7.34(0.79)$

$7.82(0.79)$

Limb strength gain $(\%)$

ean (standard deviation).

Abbreviations: CHF, chronic heart failure; NAC, N-acetyl-1-cysteine; bpm, beats per minute; and MIP, maximum inspiratory pressure.

Statistical significance: $* \mathrm{P} \leq 0.05, * * \mathrm{P} \leq 0.01$ and ${ }^{* * *} \mathrm{P} \leq 0.001$ between any group of CHF-cachexia with and without treatment and the control rats.

$\dagger \mathrm{P} \leq 0.05, \dagger \dagger \mathrm{P} \leq 0.01$ and $\dagger \dagger \dagger \mathrm{P} \leq 0.001$ between any of the pharmacologically treated groups of rats with cachexia and CHF-cachexia animals. 
Table 2. Structural characteristics of the gastrocnemius and diaphragm muscles in all animals groups

\begin{tabular}{|c|c|c|c|c|}
\hline & $\begin{array}{c}\text { Control } \\
n=10 \\
\end{array}$ & $\begin{array}{c}\text { CHF-cachexia } \\
n=10\end{array}$ & $\begin{array}{c}\text { CHF-cachexia } \\
+ \text { NAC } \\
n=10 \\
\end{array}$ & $\begin{array}{c}\text { CHF-cachexia } \\
+ \text { Bortezomib } \\
n=10 \\
\end{array}$ \\
\hline \multicolumn{5}{|l|}{ Diaphragm } \\
\hline \multicolumn{5}{|l|}{ Muscle fiber type, $\%$} \\
\hline Type I fibers & $27.79(6.60)$ & $27.46(3.53)$ & $29.63(3.77)$ & $27.57(4.03)$ \\
\hline Type II fibers & $72.21(6.60)$ & $72.54(3.53)$ & $70.37(3.77)$ & $72.43(4.03)$ \\
\hline \multicolumn{5}{|l|}{ Muscle fiber size (CSA), $\mu \mathrm{m}^{2}$} \\
\hline Cross-sectional area, type I fibers & $748(81)$ & $583(116)^{* *}$ & $683(48) \dagger$ & $720(79) \dagger \dagger$ \\
\hline Cross-sectional area, type II fibers & $697(126)$ & $493(54) * * *$ & $703(81) \dagger \dagger \dagger$ & $860(52)^{*}, \dagger \dagger \dagger$ \\
\hline Total muscle structural abnormalities, $\%$ & $1.96(0.83)$ & $5.18(0.51)^{* * *}$ & $3.73(0.95) \dagger \dagger$ & $4.22(0.37)^{* * *}, \dagger$ \\
\hline Internal nuclei, $\%$ & $0.59(0.25)$ & $1.27(0.41)^{* * *}$ & $1.07(0.53)$ & $1.88(0.68)^{* * *}$ \\
\hline Inflammatory cells, $\%$ & $1.22(0.55)$ & $3.59(0.71)^{* * *}$ & $2.66(0.42)^{* *}, \dagger$ & $2.24(0.45)^{*}, \dagger \dagger$ \\
\hline Other items $(\S), \%$ & $0.16(0.14)$ & $0.32(0.16)^{*}$ & $0.00(0) \dagger$ & $0.09(0.1) \dagger$ \\
\hline Total leukocytes + macrophages cells / area $\left(u^{2}\right)$ & $0.08(0.09)$ & $0.29(0.14)^{* *}$ & $0.07(0.05) \dagger$ & $0.1(0.12) \dagger$ \\
\hline TUNEL-positive nuclei, $\%$ & $57.61(9.1)$ & $72.74(10.1)^{* *}$ & $51.89(6.9) \dagger \dagger$ & $52.96(11.2) \dagger$ \\
\hline \multicolumn{5}{|l|}{ Gastrocnemius } \\
\hline \multicolumn{5}{|l|}{ Muscle fiber type, $\%$} \\
\hline Type I fibers & $28.68(4.33)$ & $27.17(4.53)$ & $28.01(4.00)$ & $25.59(2.43)$ \\
\hline Type II fibers & $71.32(4.33)$ & $72.83(4.53)$ & $70.69(4.60)$ & $74.41(2.43)$ \\
\hline \multicolumn{5}{|l|}{ Muscle fiber size (CSA), $\mu \mathrm{m}^{2}$} \\
\hline Cross-sectional area, type I fibers & $1431(384)$ & $1129.07(146)^{*}$ & $1446.36(200) \dagger$ & $1874(554) \dagger \dagger \dagger$ \\
\hline Cross-sectional area, type II fibers & $1422(272)$ & $1106(207)^{* *}$ & $1565(333) \dagger$ & $1654(207) \dagger \dagger$ \\
\hline Total muscle structural abnormalities, $\%$ & $0.85(0.43)$ & $2.83(0.49)^{* * *}$ & $1.29(0.16) \dagger \dagger \dagger$ & $1.99(0.36)^{* * *}, \dagger$ \\
\hline
\end{tabular}


Internal nuclei, $\%$

Inflammatory cells, $\%$

Other items $(\S), \%$

Total leukocytes + macrophages cells $/$ area $\left(\mathrm{um}^{2}\right)$

TUNEL-positive nuclei, \%

$\begin{array}{cc}0.37(0.24) & 0.70(0.23)^{* *} \\ 0.44(0.30) & 1.79(0.50)^{* * *} \\ 0.44(0.42) & 0.34(0.25)^{* *} \\ 0.08(0.08) & 0.17(0.07)^{*} \\ 54.56(19.8) & 79.28(9.2)^{* *}\end{array}$

$0.37(0.24)$
$0.60(0.41)$
$0.59(0.07) \dagger \dagger$
$0.1(0.1) \dagger$
$0.09(0.05) \dagger$
$59.28(13.6) \dagger$

$1.16(0.30)^{* * *}, \dagger$

$0.82(0.43) \dagger \dagger$

$0.01(0.02) \dagger$

$0.07(0.03) \dagger$

$28.99(19.8) \dagger \dagger \dagger$

Variables are presented as mean (standard deviation).

Abbreviations: CHF, chronic heart failure; NAC, N-acetyl-1-cysteine; and CSA, cross-sectional area.

(§) Other items: Percentage of the sum of abnormal and inflammed fibers and lipofucsin.

Statistical significance: $* \mathrm{P} \leq 0.05, * * \mathrm{P} \leq 0.01$ and ${ }^{* * *} \mathrm{P} \leq 0.001$ between any group of CHF-cachexia with and without treatment and the control rats. $\dagger \mathrm{P} \leq 0.05$, $\dagger \mathrm{P} \leq 0.01$ and $\dagger \dagger \mathrm{P} \leq 0.001$ between any of the pharmacologically treated groups of rats with cachexia and CHF-cachexia animals. 
Barreiro et al. Figure 1

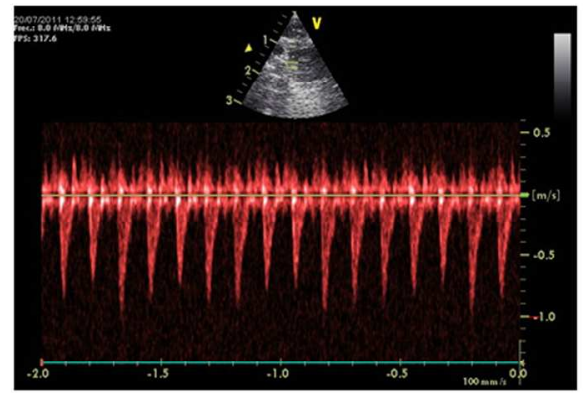

Control

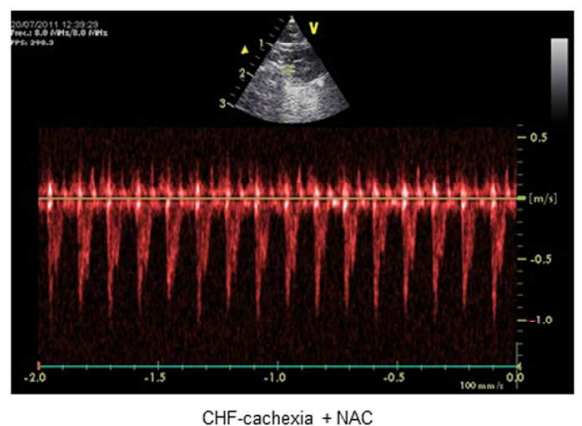

CHF-cachexia + NAC

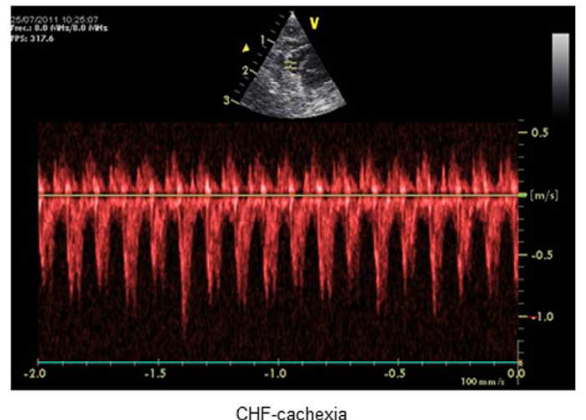

CHF-cachexia

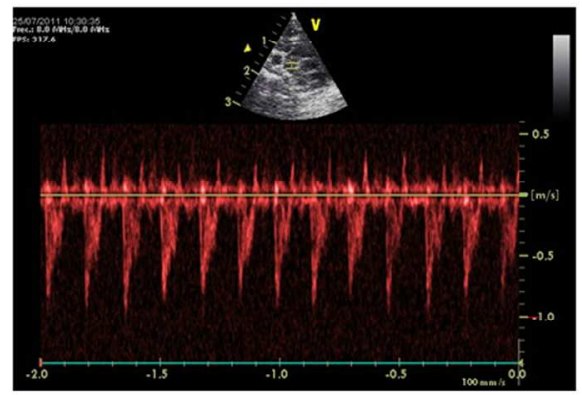

CHF-Cachexia + Bortezomib

Figure 1

$254 \times 190 \mathrm{~mm}(300 \times 300$ DPI $)$

John Wiley \& Sons, Inc. 
Barreiro et al. Figure 2

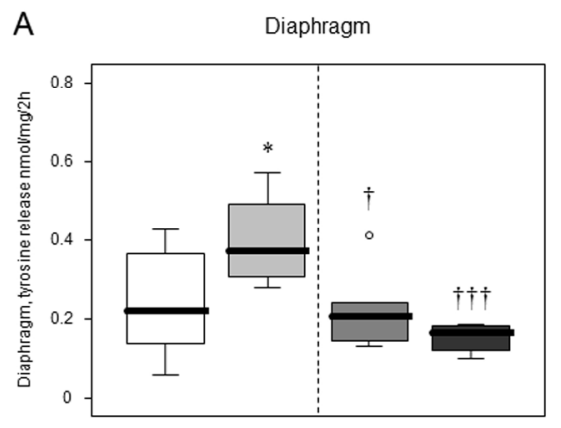

B

Gastrocnemius
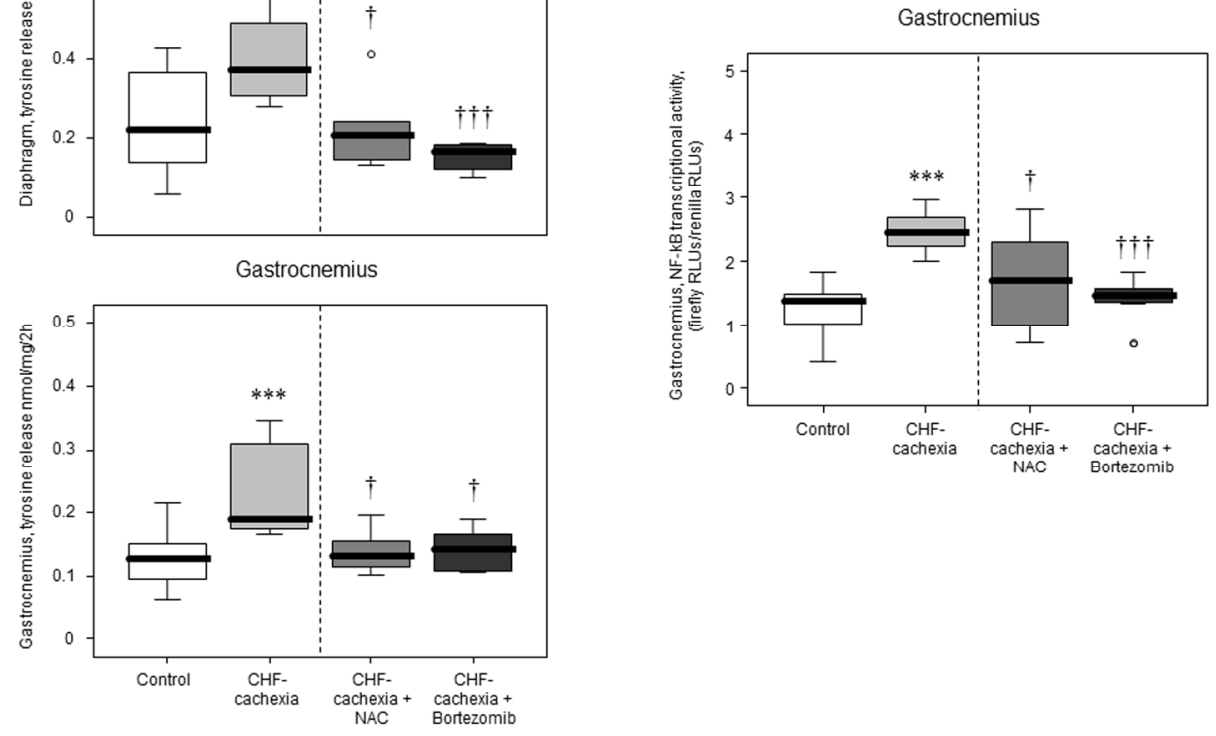

Figure $2 \mathrm{~A}-2 \mathrm{~B}$

$254 \times 190 \mathrm{~mm}$ (300 x $300 \mathrm{DPI})$

34

35

36

37

38

39

40

41

42

43

44

45

46

47

48

49

50

51

52

53

54

55

56

57

58

59

60

John Wiley \& Sons, Inc. 
Barreiro et al. Figure 2C

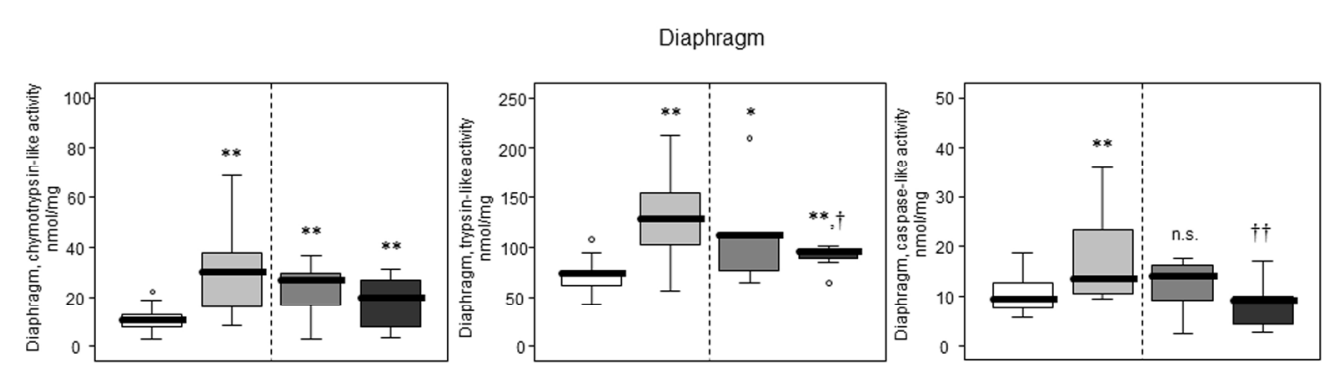

Gastrocnemius
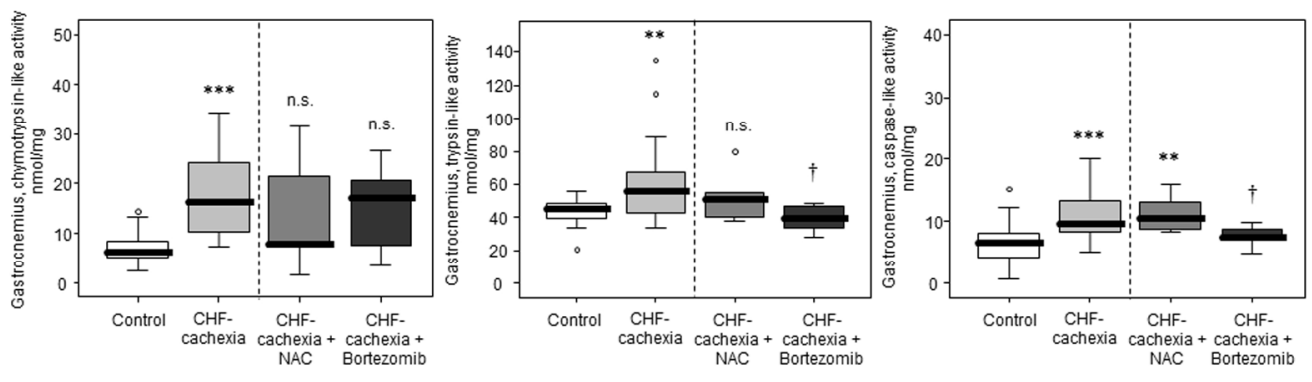

Figure 2C

$254 \times 190 \mathrm{~mm}$ ( $300 \times 300$ DPI)

36

37

38

39

40

41

42

44

45

46

47

48

49

51

52

53

54

55 
Figure $3 \mathrm{~A}-3 \mathrm{~B}$

$254 \times 190 \mathrm{~mm}(300 \times 300 \mathrm{DPI})$

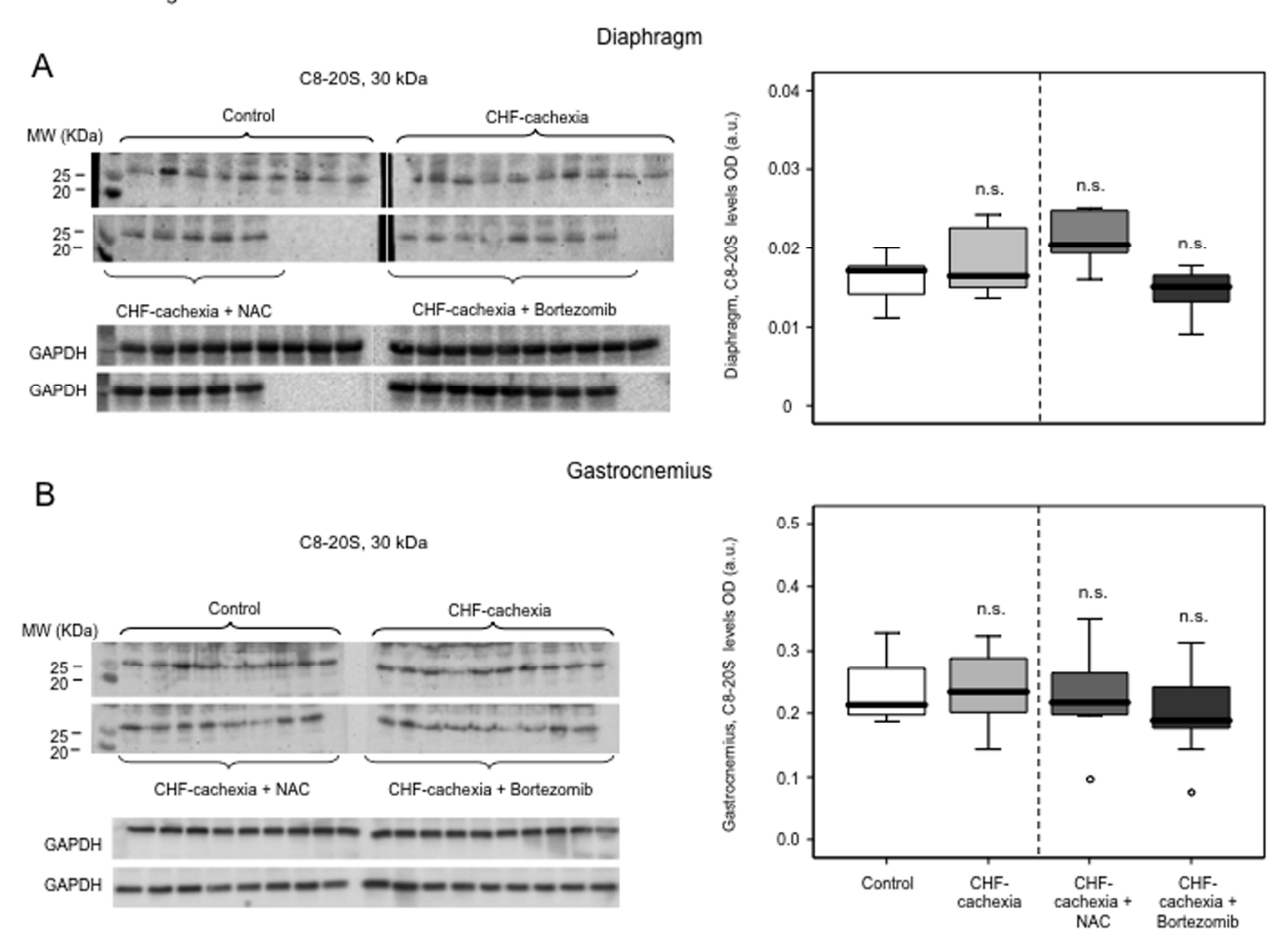


Figure $3 \mathrm{C}-3 \mathrm{D}$

$254 \times 190 \mathrm{~mm}(300 \times 300 \mathrm{DPI})$
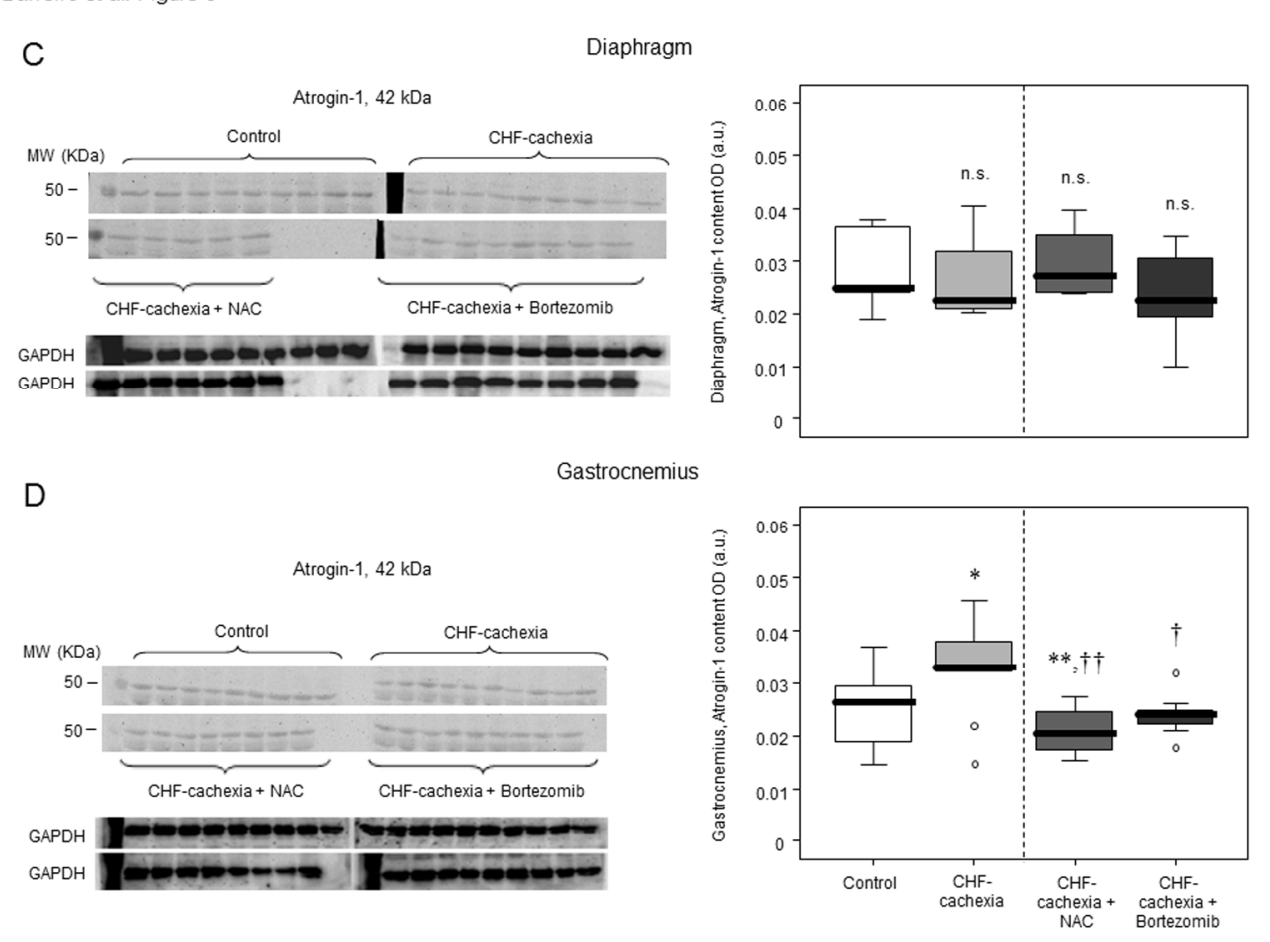

John Wiley \& Sons, Inc. 
Barreiro et al. Figure 3
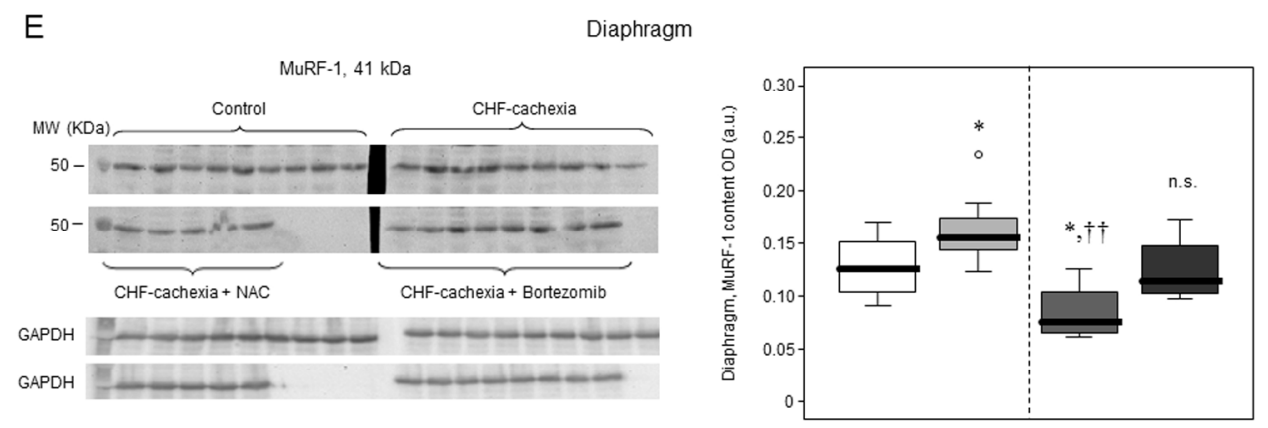

F

Gastrocnemius
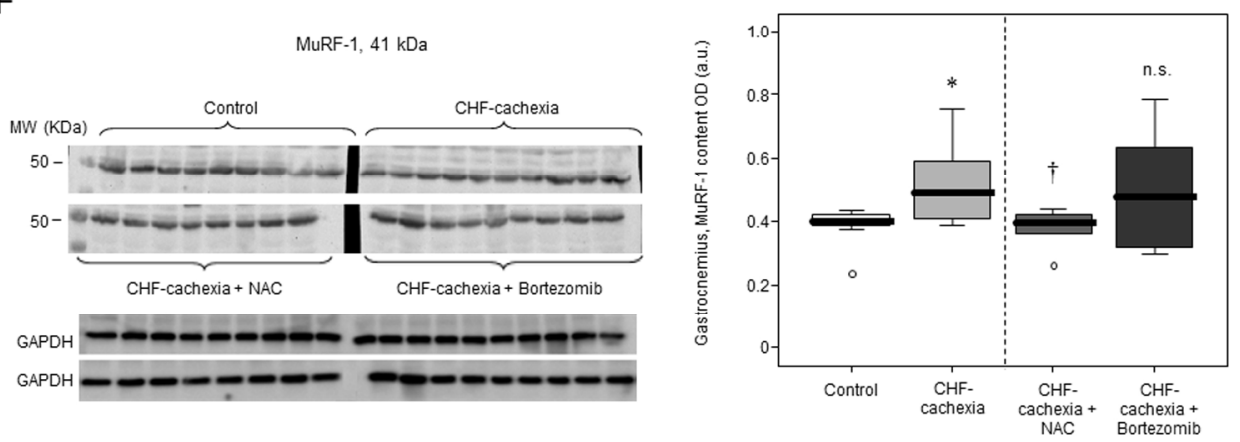

Figure $3 \mathrm{E}-3 \mathrm{~F}$ $254 \times 190 \mathrm{~mm}(300 \times 300 \mathrm{DPI})$

John Wiley \& Sons, Inc. 
Figure $3 \mathrm{G}-3 \mathrm{H}$ $254 \times 190 \mathrm{~mm}(300 \times 300$ DPI $)$

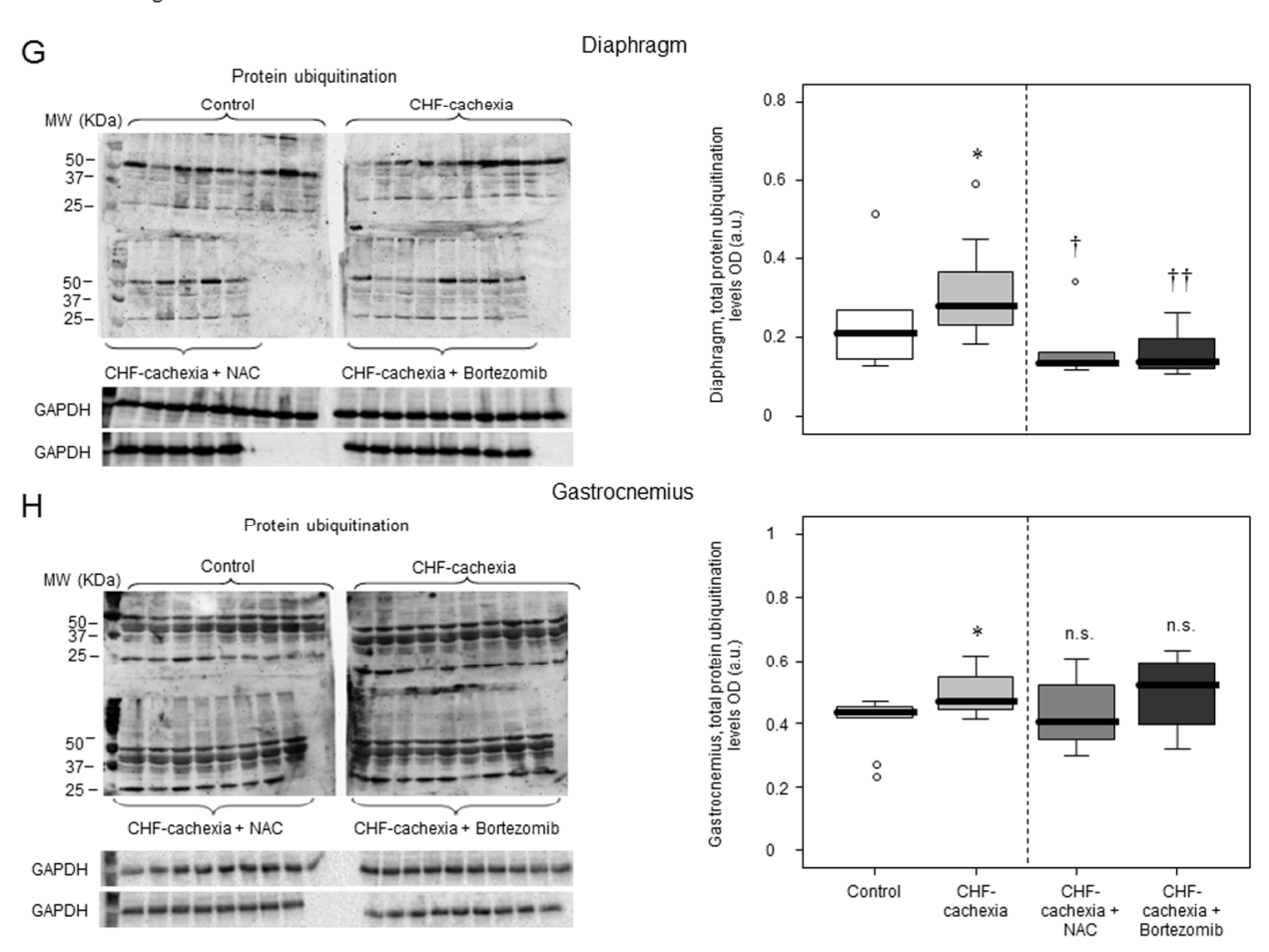


Barreiro et al. Figure 4A
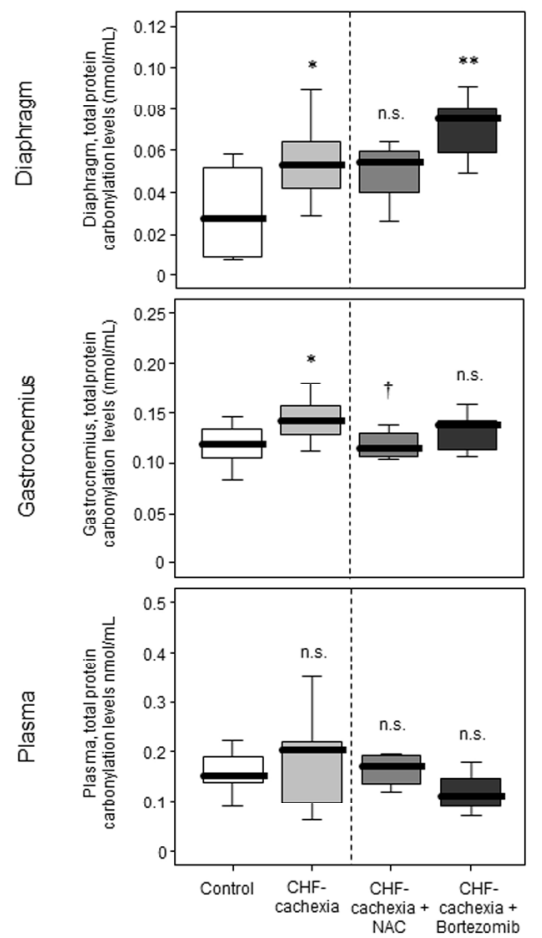

Figure $4 \mathrm{~A}$

$254 \times 190 \mathrm{~mm}(300 \times 300 \mathrm{DPI})$

35

36

37

38

39

40

41

42

43

44

45

46

47

48

49

50

51

52

53

54

55

56

57

58

59

60

John Wiley \& Sons, Inc. 
Barreiro et al. Figure 4
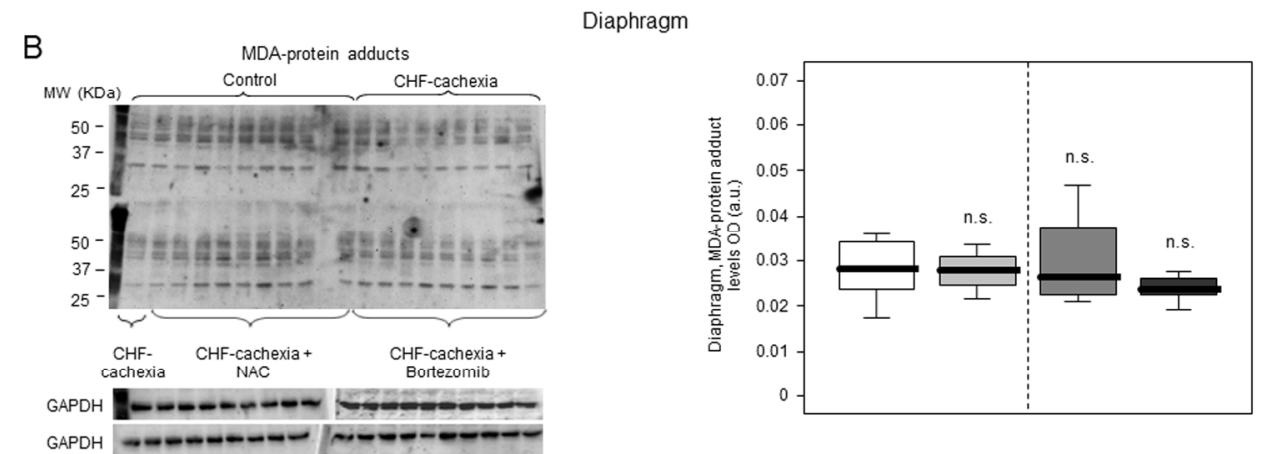

C
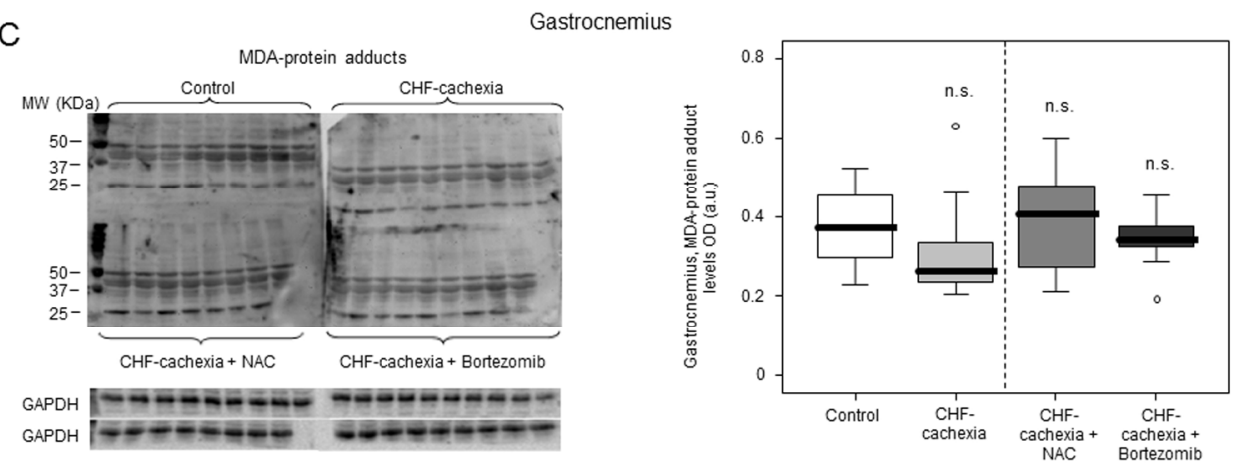

Figure 4B-4C

$254 \times 190 \mathrm{~mm}$ (300 x 300 DPI)

John Wiley \& Sons, Inc. 
Barreiro et al. Figure 4

D

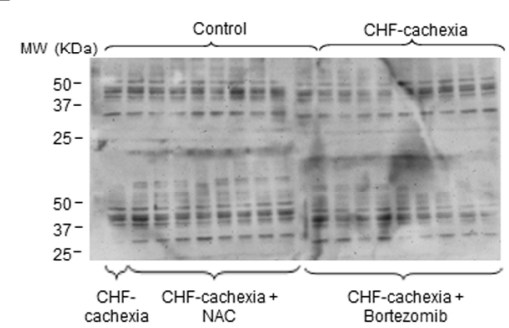

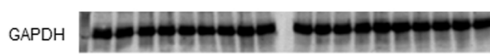

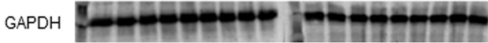

E
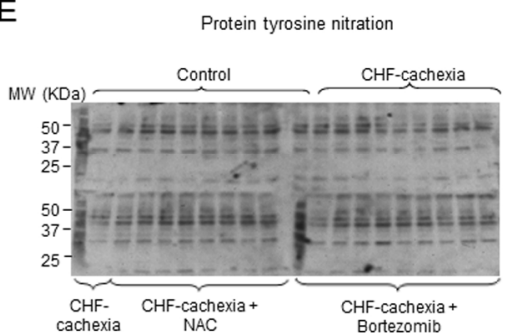

GAPDH 7 -

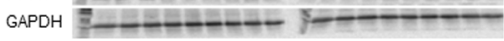

Diaphragm

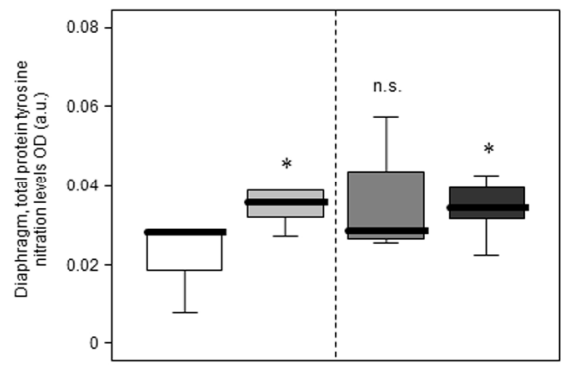

Gastrocnemius

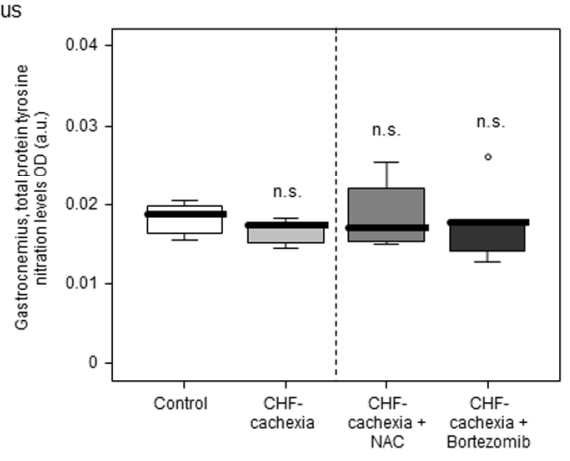

Figure 4D-4E $254 \times 190 \mathrm{~mm}(300 \times 300 \mathrm{DPI})$

John Wiley \& Sons, Inc. 
Figure $4 \mathrm{~F}-4 \mathrm{G}$ $254 \times 190 \mathrm{~mm}(300 \times 300 \mathrm{DPI})$
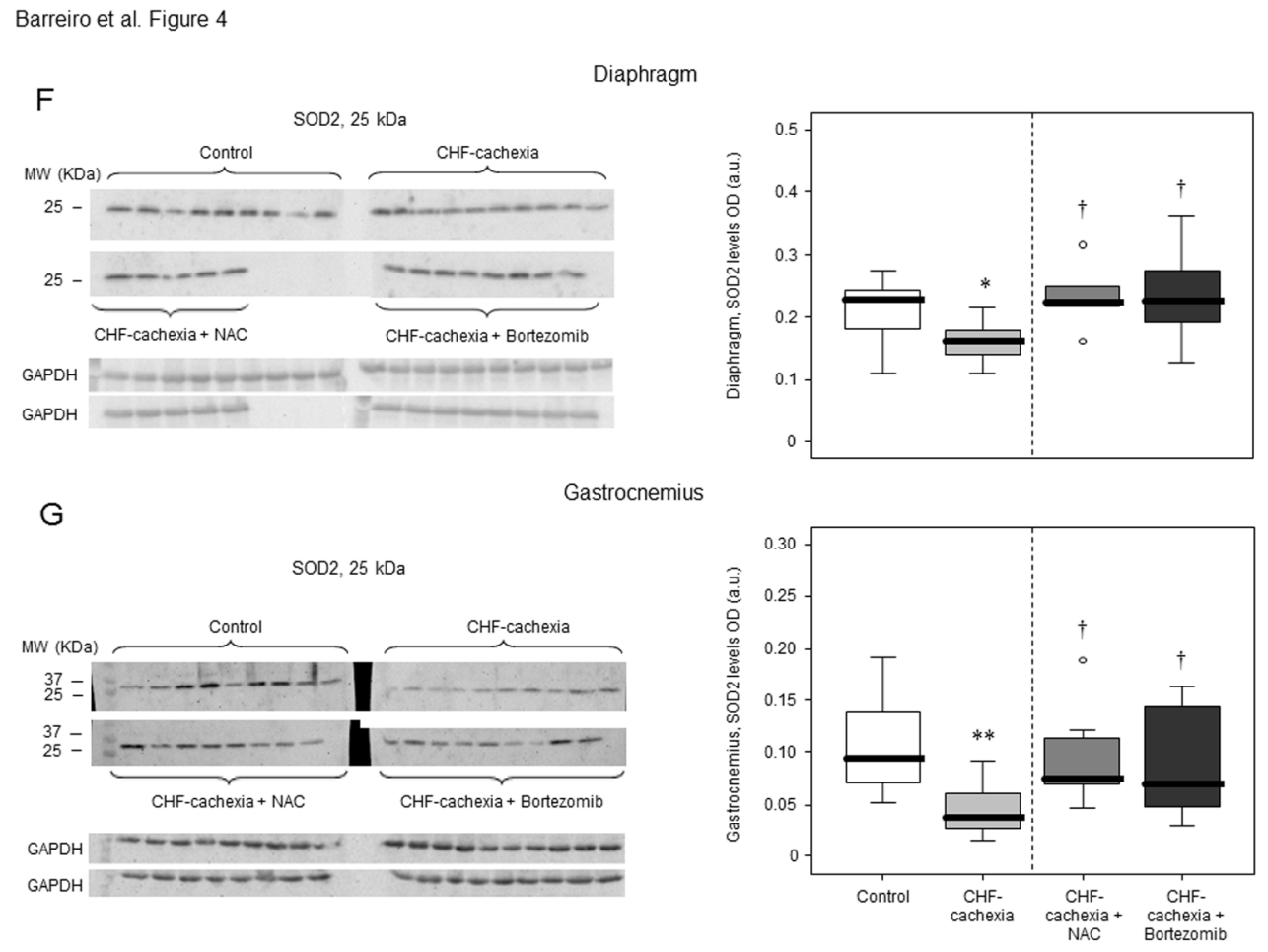

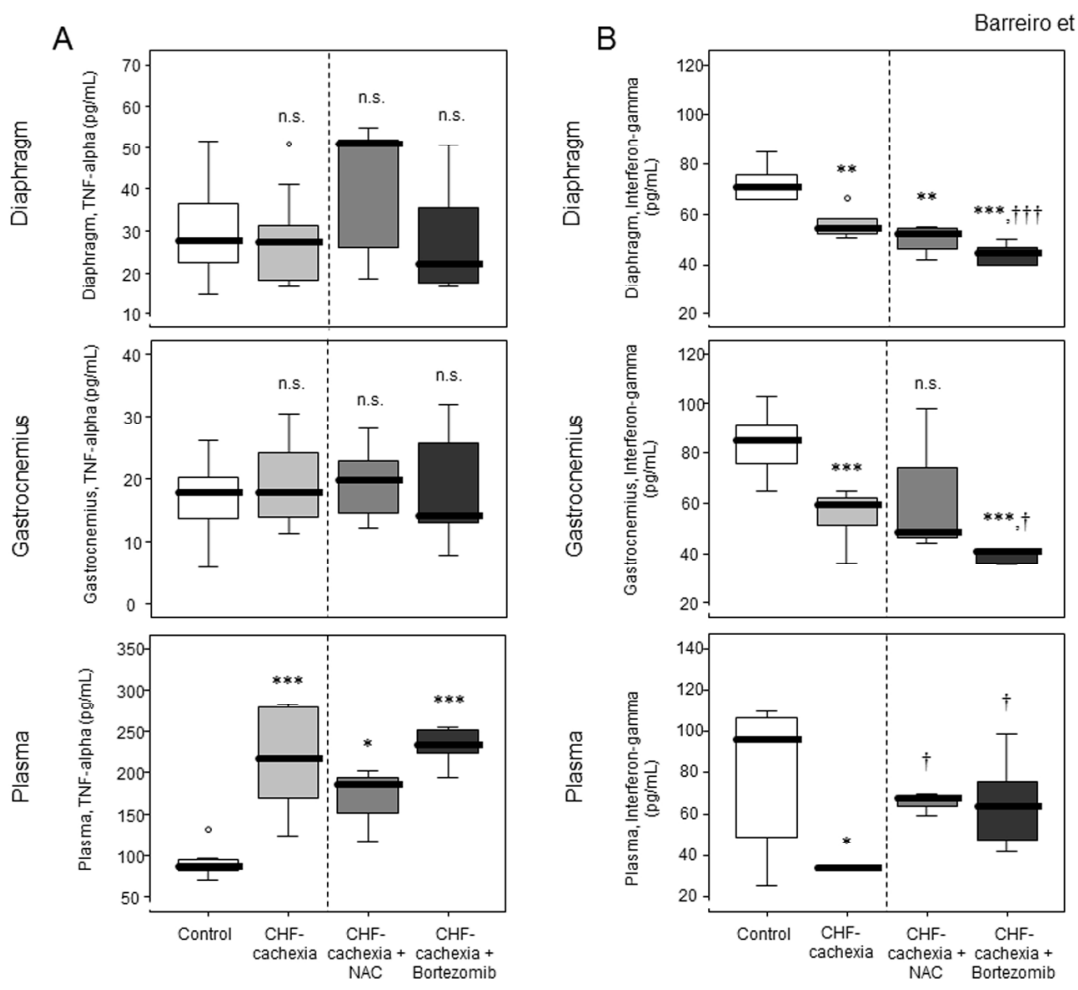

Figure $5 \mathrm{~A}-5 \mathrm{~B}$

$254 \times 190 \mathrm{~mm}$ (300 x 300 DPI)

34

35

36

37

38

39

40

41

42

43

44

45

46

47

48

49

50

51

52

53

54

55

56

57

58

59

60

John Wiley \& Sons, Inc. 

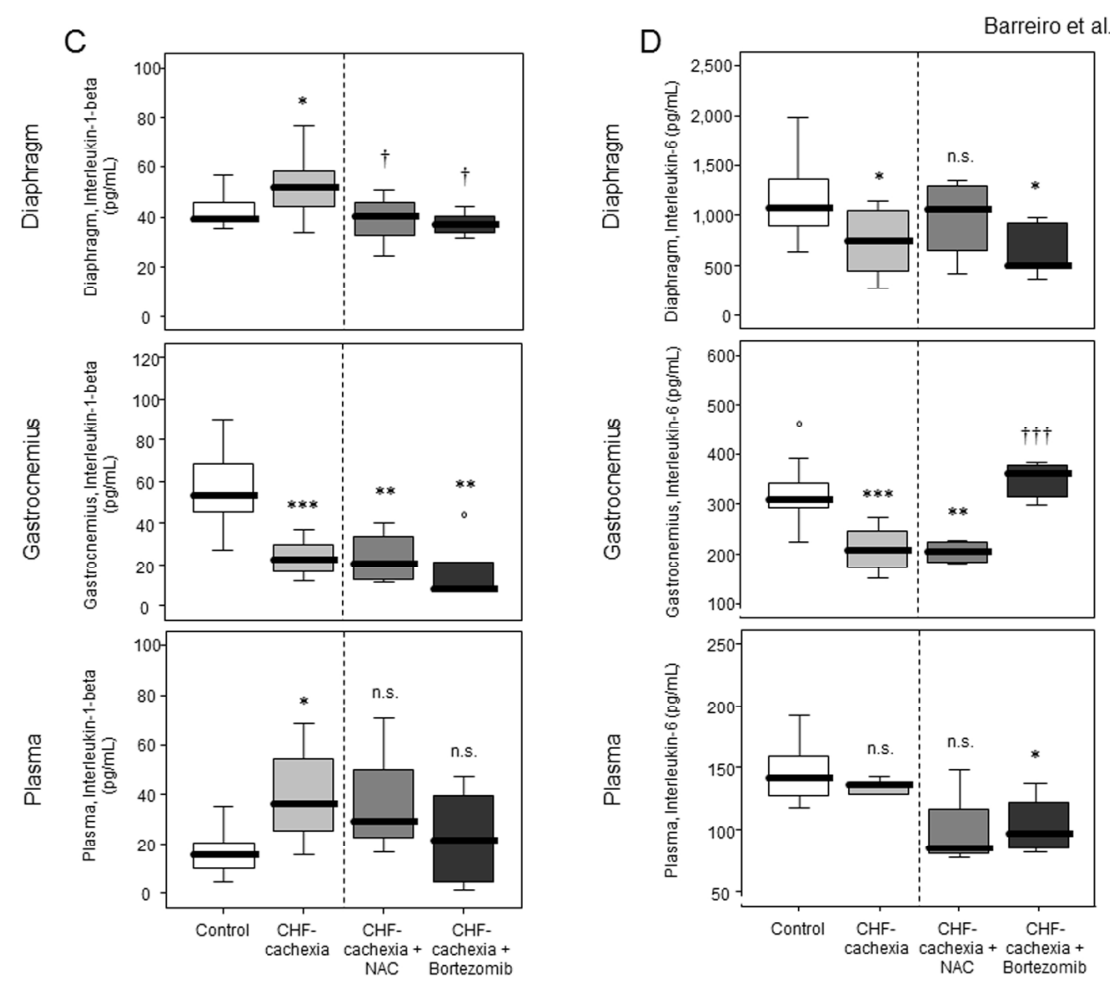

Figure 5C-5D

$254 \times 190 \mathrm{~mm}(300 \times 300 \mathrm{DPI})$

John Wiley \& Sons, Inc. 
Barreiro et al. Figure $6 \mathrm{~A}$
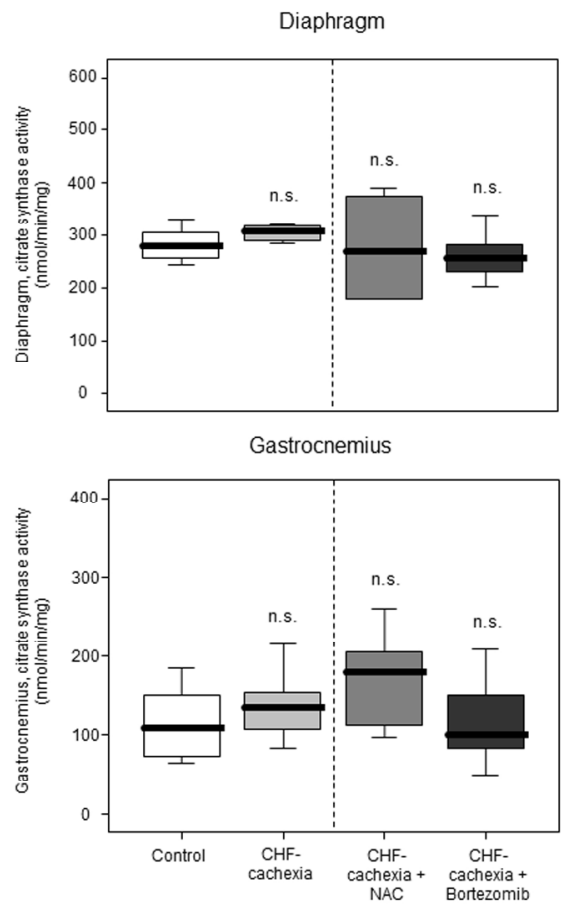

Figure $6 \mathrm{~A}$

$254 \times 190 \mathrm{~mm}(300 \times 300 \mathrm{DPI})$

34

35

36

37

38

39

40

41

42

43

44

45

46

47

48

49

50

51

52

53

54

55

56

57

58

59

60

John Wiley \& Sons, Inc. 
Figure 6B
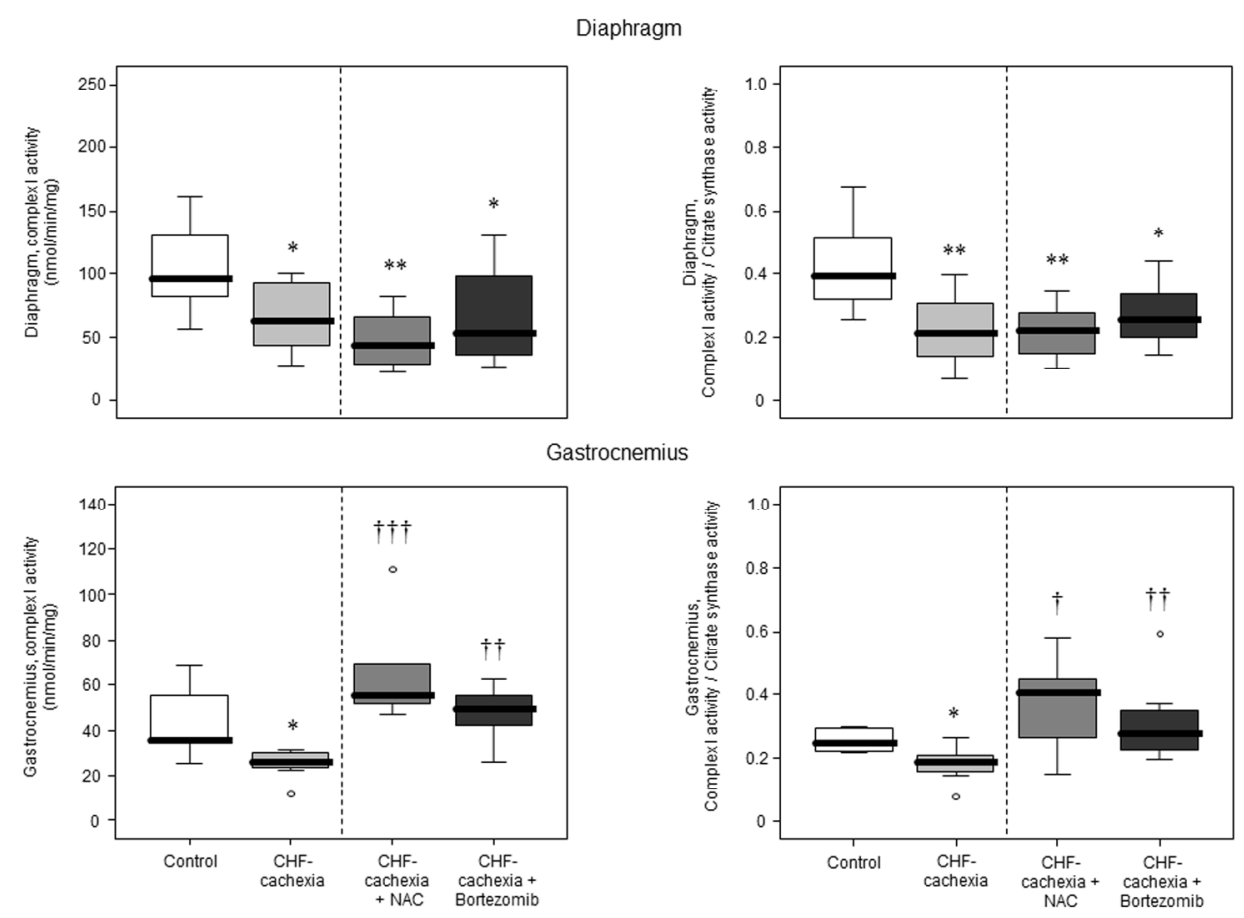

\section{$254 \times 190 \mathrm{~mm}$ (300 x 300 DPI)}

John Wiley \& Sons, Inc. 
Barreiro et al. Figure $6 \mathrm{C}$
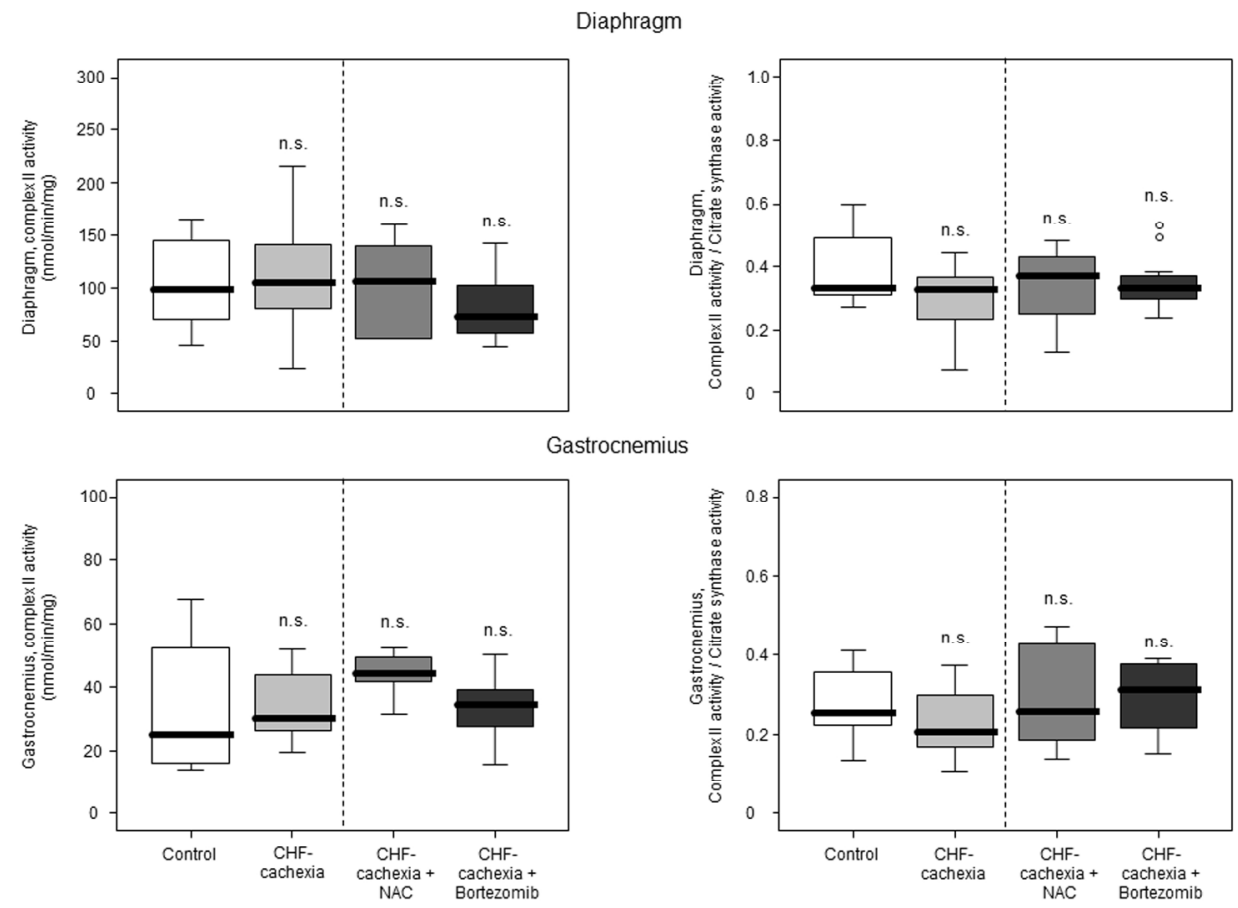

Figure 6C

$254 \times 190 \mathrm{~mm}(300 \times 300$ DPI $)$

34

35

36

37

38

39

40

41

42

43

44

45

46

47

48

49

50

51

52

53

54

55

56

57

58

59

60

John Wiley \& Sons, Inc. 
Figure 6D

$254 \times 190 \mathrm{~mm}(300 \times 300$ DPI $)$
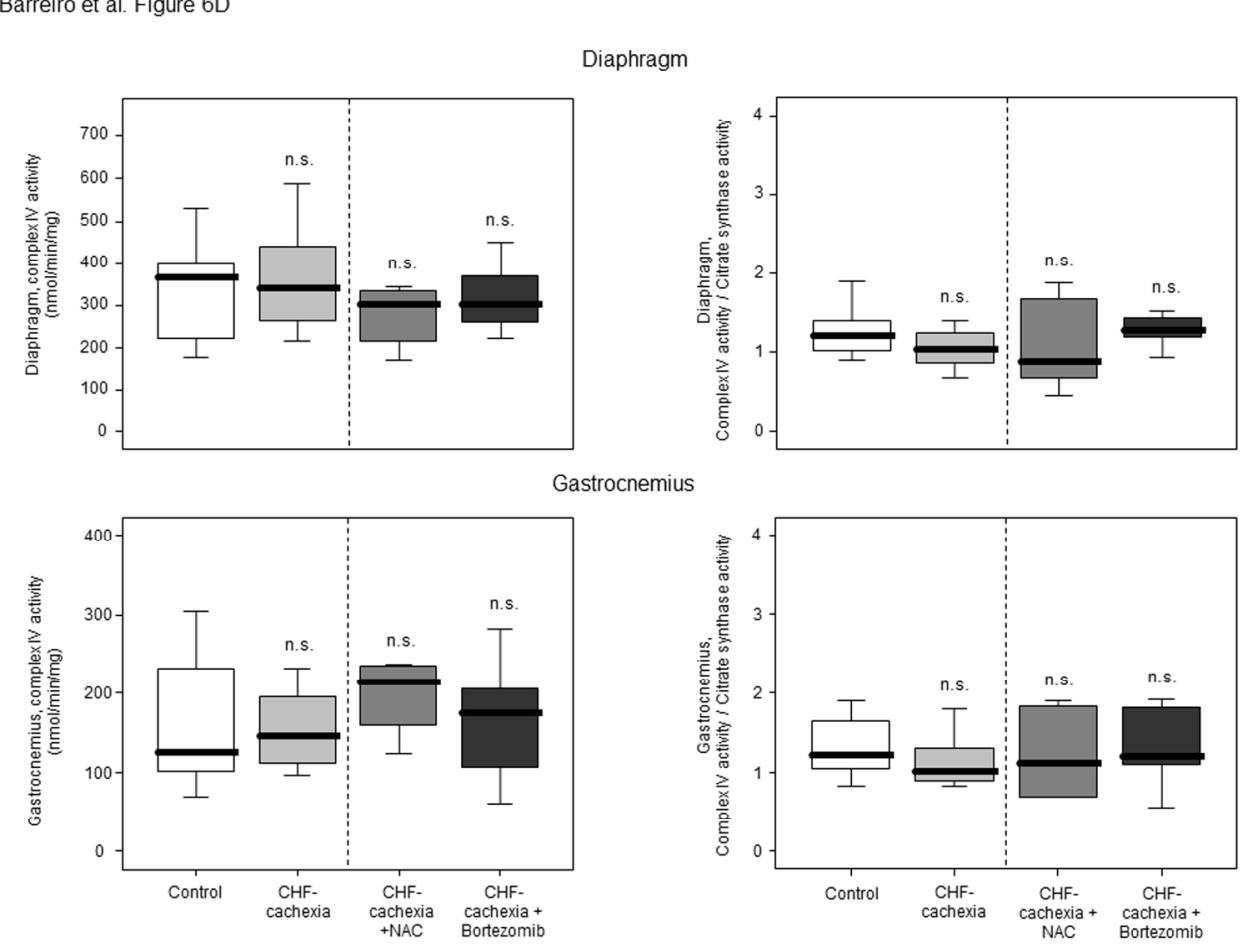

John Wiley \& Sons, Inc. 
Figure 6E $254 \times 190 \mathrm{~mm}(300 \times 300 \mathrm{DPI})$

John Wiley \& Sons, Inc. 
1

2

3

4

5

6

7

8

9

10

11

12

13

14

15

16

17

18

19

20

21

22

23

24

25

26

27

28

29

30

31

32

33

34

35

36

37

38

39

40

41

42

43

44

45

46

47

48

49

50

51

52

53

54

55

56

57

58

59

60
Barreiro et al. Figure $6 \mathrm{~F}$
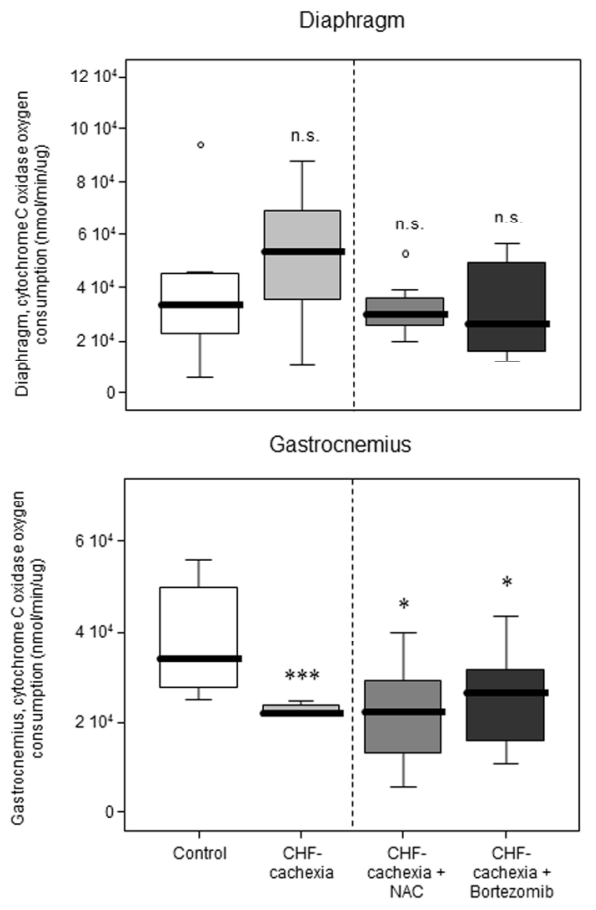

Figure $6 \mathrm{~F}$

$254 \times 190 \mathrm{~mm}(300 \times 300$ DPI $)$

John Wiley \& Sons, Inc. 
Figure $7 \mathrm{~A}-7 \mathrm{~B}$

$254 \times 190 \mathrm{~mm}(300 \times 300 \mathrm{DPI})$

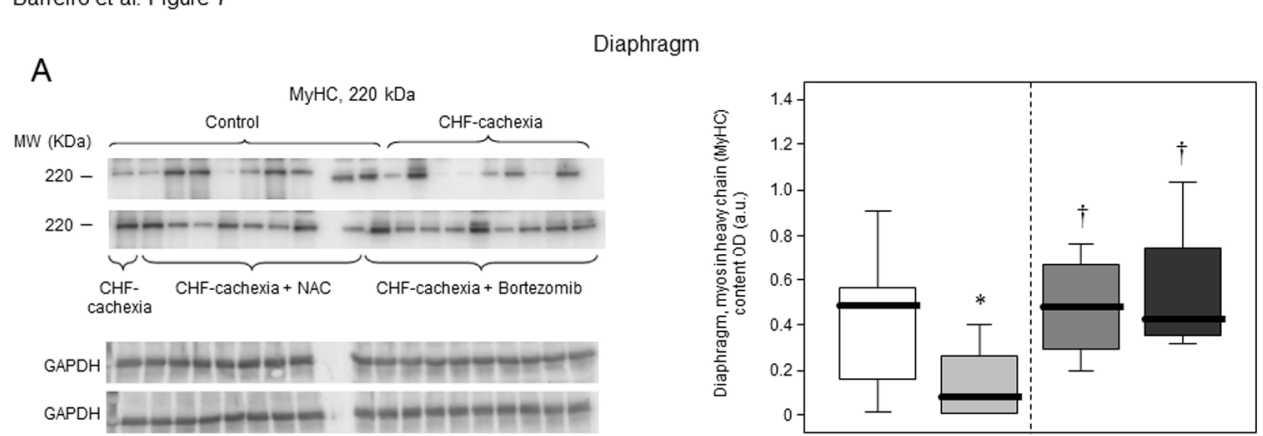

B
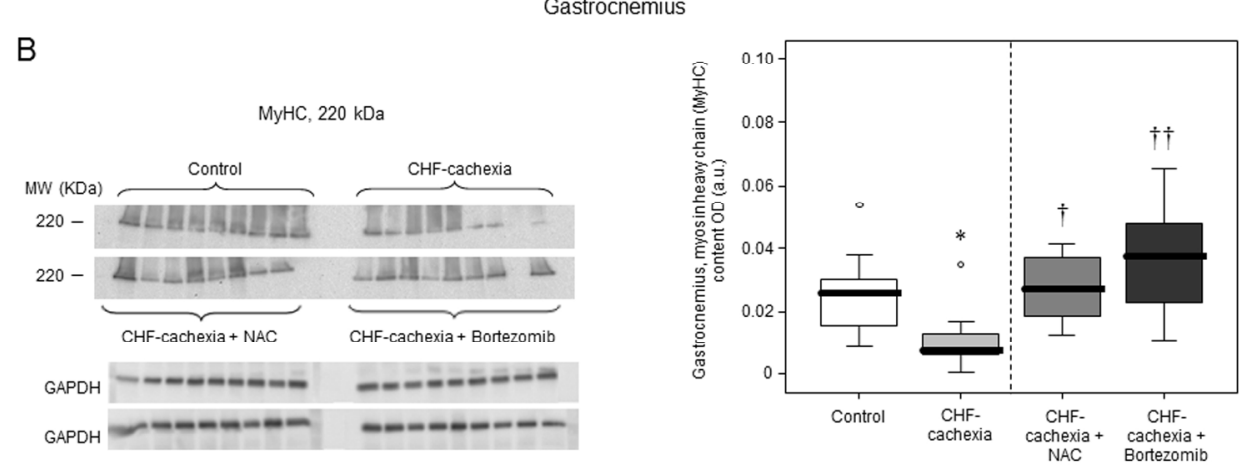

Gastrocnemius

John Wiley \& Sons, Inc. 
Barreiro et al. Figure 7C
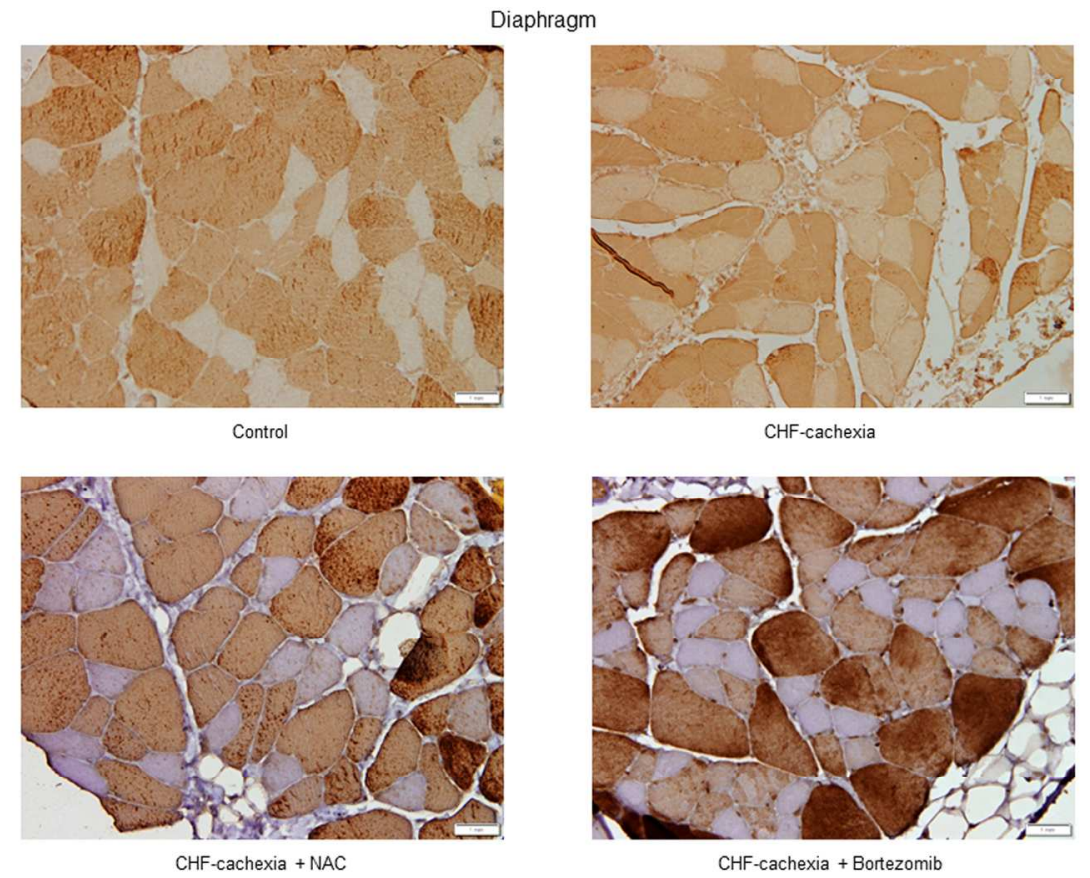

Figure $7 \mathrm{C}$

$254 \times 190 \mathrm{~mm}$ ( $300 \times 300$ DPI)

35

36

37

38

39

40

41

42

44

45

46

47

48

49

51

52

53

54

55

John Wiley \& Sons, Inc. 
Barreiro et al. Figure 7D

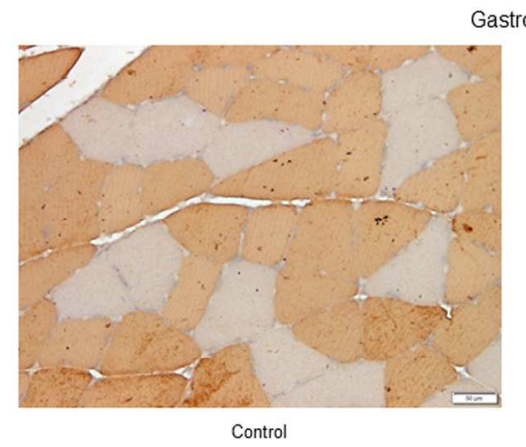

Gastrocnemius

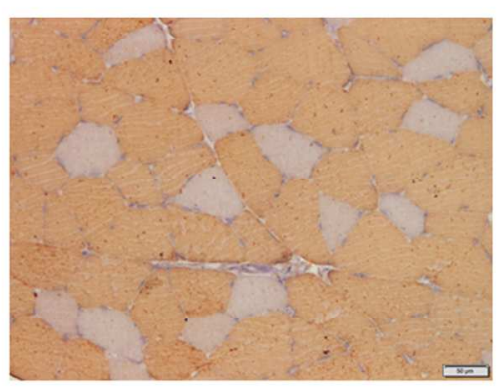

CHF-cachexia + NAC

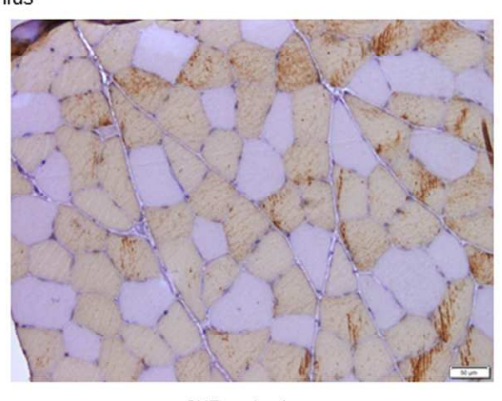

CHF-cachexia

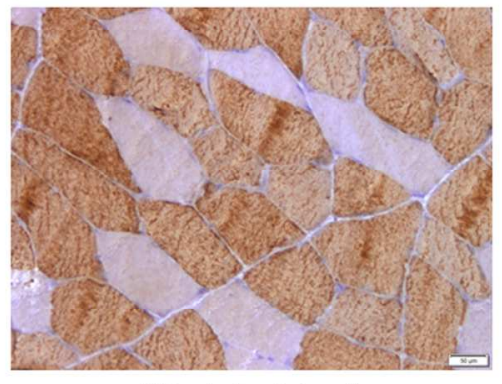

CHF-cachexia + Bortezomib

Figure 7D

$254 \times 190 \mathrm{~mm}$ (300 x 300 DPI)

John Wiley \& Sons, Inc. 
Barreiro et al. Figure 7E
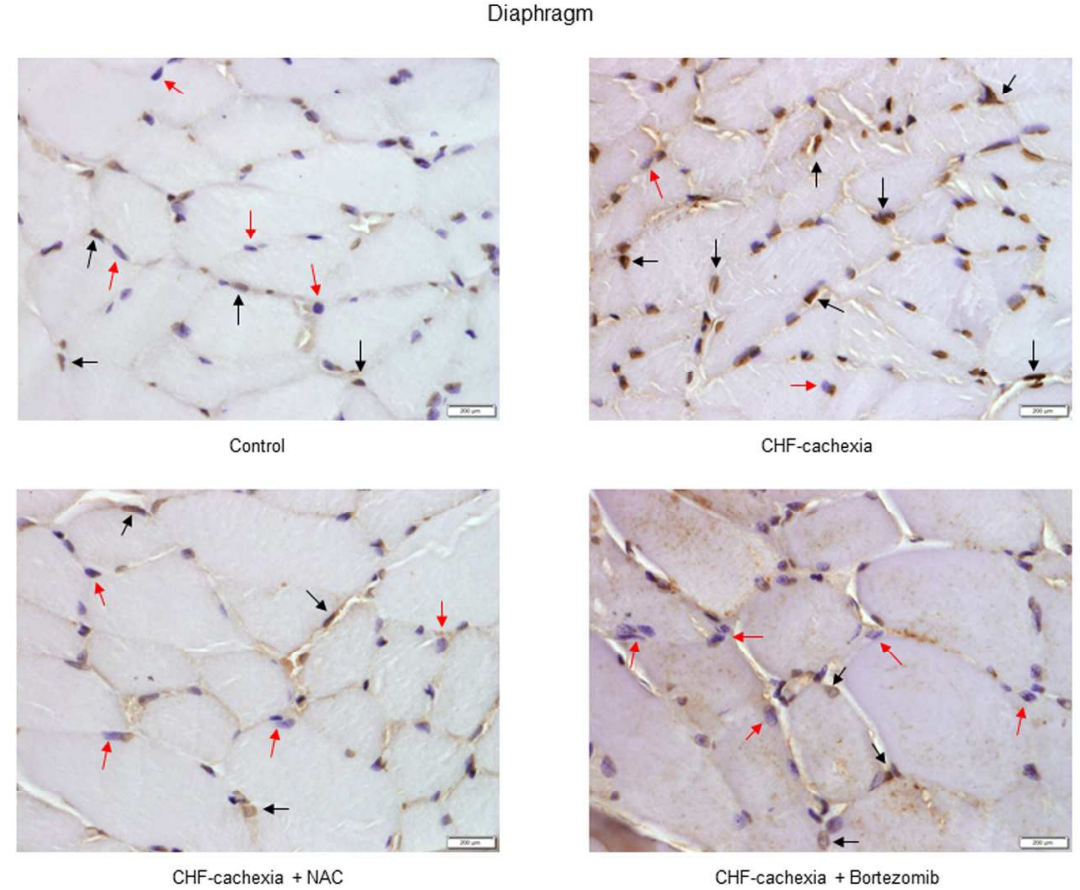

CHF-cachexia + Bortezomib

Figure 7E

$254 \times 190 \mathrm{~mm}$ (300 x 300 DPI)

John Wiley \& Sons, Inc. 
Barreiro et al. Figure 7F

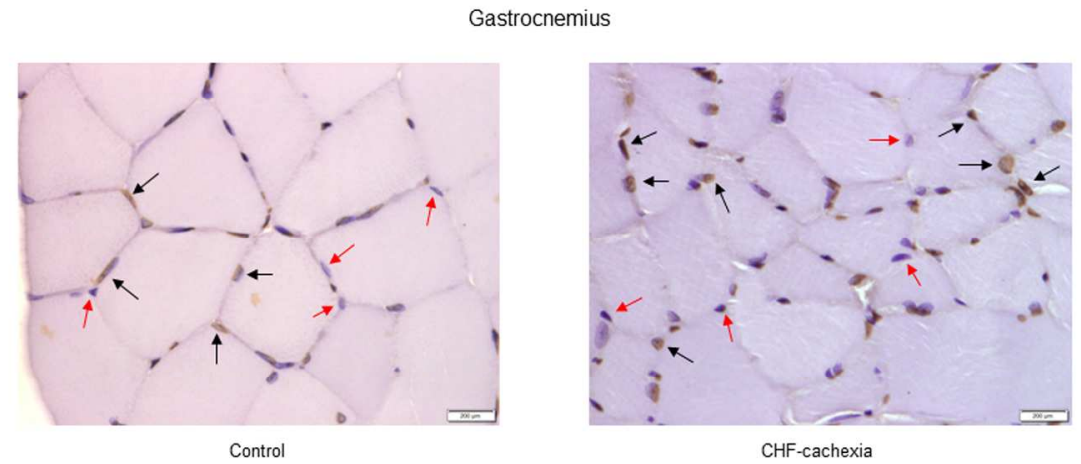

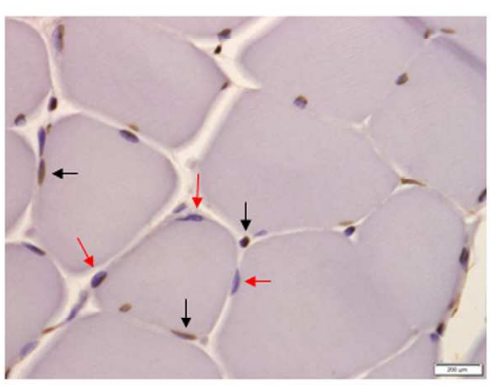

CHF-cachexia + NAC

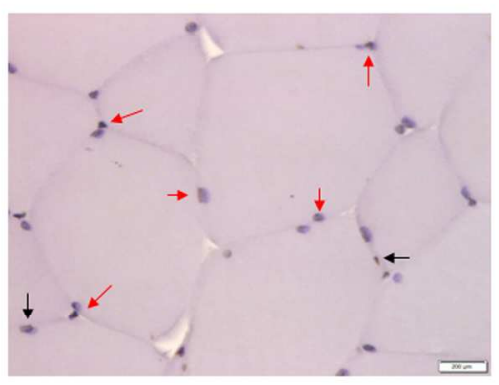

CHF-cachexia + Bortezomib

Figure 7F

$254 \times 190 \mathrm{~mm}$ ( $300 \times 300$ DPI) 